\title{
A continuous latitudinal energy balance model to explore non-uniform climate engineering strategies
}

\author{
Federica Bonetti $^{1}$ (D) $\cdot$ Colin Mclnnes $^{1}$
}

Received: 7 December 2017 / Accepted: 27 September 2018 / Published online: 6 October 2018

(c) The Author(s) 2018

\begin{abstract}
In order to investigate the effects of solar radiation management (SRM) technologies for climate engineering, an analytical model describing the main latitudinal dynamics of the Earth's climate with closed-loop control has been developed. The model is a time-dependent Energy Balance Model (EBM) with latitudinal resolution and allows for the evaluation of nonuniform climate engineering strategies. The resulting partial differential equation is solved using a Green's function approach. This model offers an efficient analytical approach to design strategies that counteract climate change on a latitudinal basis to overcome regional disparities in cooling. Multi-objective analyses are considered and time-dependent analytical expressions of control functions with latitudinal resolution can be obtained in several circumstances. Results broadly comparable with the literature are found, demonstrating the utility of the model in rapidly assessing new climate engineering controls laws and strategies. For example, the model is also used to quickly assess the trade-off between the number of degrees of freedom of SRM and the rms error in latitudinal temperature compensation. Moreover, using the EBM the dynamics of the ice line can be investigated and a Lyapunov stability analysis is employed to estimate the maximum reduction of solar insolation through climate engineering before the current climate falls into an ice-covered state. This provides an extreme operational boundary to future climate engineering ventures.
\end{abstract}

Keywords SRM $\cdot$ Climate engineering $\cdot$ Climate modelling $\cdot$ Multi-objective control $\cdot$ Ice line $\cdot$ Lyapunov stability

\section{Introduction}

Climate engineering Blackstock et al. (2009) aims to partly offset the impacts of human-driven climate change. It involves techniques developed both to reduce the concentration of carbon dioxide in the atmosphere [Carbon Dioxide Removal (CDR) methods] and to counteract the radiative forcing generated [Solar Radiation Management (SRM) methods] Vaughan and Lenton (2011). High-speed computers and high-fidelity numerical models for the climate system can be used to evaluate climate engineering strategies. For example, General Circulation Models (GCMs) have many millions of degrees of freedom and, although they can be physically realistic, these models are computationally

Federica Bonetti

F.Bonetti.1@research.gla.ac.uk

Colin McInnes

Colin.McInnes@glasgow.ac.uk

1 School of Engineering, University of Glasgow, Glasgow G128QQ, Scotland expensive. In contrast, low order one-dimensional climate models can be found in Budyko (1969), Sellers (1969) and North et al. (1981). In particular, considering elementary thermodynamics, Budyko (1969) and Sellers (1969) proposed low order climate models to investigate the climate state as a function of the solar constant, whereas North et al. (1981) investigated in detail global energy balance models using a general transport term. Moreover, a Green's function approach is used in North et al. (1981) to obtain the explicit analytical solution of a diffusive climate model in terms of hypergeometric functions.

This paper aims to approximate the complexity of the climate system with a simple model that takes into account its main features as described in North et al. (1981), and to develop continuous control laws for climate engineering as a function of latitude. Therefore, efforts have been made to develop a continuous-time PDE system with latitudinal resolution with which it is possible to explore control strategies to begin to investigate issues related to regional disparities and the side effects of SRM techniques. This continuous PDE model extends a simple 3 box model which has been 
used to investigate the use of adaptive control for climate engineering Bonetti and McInnes (2018).

The use of efficient analytic methods provides a useful tool to rapidly asses SRM strategies with latitudinal resolution and allows efficient application of multi-objective analyses. Moreover, through this procedure the latitudinal impact of SRM can be directly addressed. In general, the pattern of insolation reduction that can be generated using SRM does not match the pattern of climate change impacts due to increased forcing due to $\mathrm{CO}_{2}$. In the literature regional disparities due to SRM have been widely discussed (Heyen et al. 2015; Moreno-Cruz et al. 2012). In MacMartin et al. (2012) and Kravitz et al. (2016) an atmosphere-ocean general circulation model (AOGCM) is used to explore the potential of SRM with multiple degrees of freedom. Also, in [9], Kravitz et al. (2017), MacMartin et al. (2017), injections of sulphate aerosols at multiple locations are considered to counteract incoming solar radiation with a coupled atmosphere-ocean general circulation model.

Despite that the analytical model developed in this paper is a simplification of other more realistic numerical models, broadly comparable results are found. This demonstrates the utility of the model in rapidly assessing new climate engineering strategies and controls laws. Again, the model can assess the trade-off between the number of degrees of freedom of SRM and the RMS error in latitudinal temperature compensation, for example.

Constraints on SRM are also explored through ice line dynamics, providing extreme operational limits on SRM obtained through the latitudinal model. This analysis illustrates the scale of the required SRM manipulation and insolation reduction that would be required to trigger an instability of the climate system, demonstrating that this limit is far beyond expected SRM interventions. With respect to other similar calculations in the literature (Coakley 1979; North et al. 1981; Schneider and Gal-Chen 1973), the insolation reduction that would trigger climate instability is given as a function of both latitude and time, providing more insight for appropriate comparisons with commonly considered SRM strategies. Moreover, the time-dependence of the model employed, and of the control function for insolation reduction, produces results which are less sensitive to changes in the solar constant Schneider and Gal-Chen (1973). This is a fundamental issue with the dynamics of the ice line and for SRM investigations in general.

Section 2.1 addresses a mathematical model to describe the climate system with a single partial differential equation (PDE); here, the analytical solution to compute the temperature perturbation due to atmospheric carbon dioxide $\left(\mathrm{CO}_{2}\right)$ is developed. Then, considering the deployment of climate engineering through a reduction of incoming solar radiation, a control law to drive the temperature perturbation to zero is developed. In Sect. 3 a multi-objective control analysis is undertaken with a PI feedback control. In Sect. 4 the PDE model is employed to find an analytical control law to achieve a desired temperature profile under a doubling of $\mathrm{CO}_{2}$ and in Sect. 5 constraints on SRM are investigated by exploring the effect of climate engineering on the dynamics of the ice line.

\section{Model and methodology}

\subsection{PDE model for the climate system}

In this section a dynamical model of the climate system with latitudinal resolution is developed. With respect to North et al. (1981), where a one-dimensional equilibrium model with diffusive heat transfer was developed to investigate ice feedback mechanisms, here temperature is also considered as a function of time. Moreover, both hemispheres are considered and differences in land and oceans are taken into account for the computation of the heat capacity for the northern and southern hemispheres. The model is then employed to explore control strategies based on a reduction of incoming solar radiation.

An Energy Balance Model (EBM) McGuffie and Henderson-Sellers (2005) is used to describe the main dynamics of the Earth's climate and the diffusion of heat between latitudinal bands. This allows for an evaluation of non-uniform climate engineering strategies. In particular, the model has the advantage of being analytically tractable, allowing new strategies to be efficiently assessed prior to more detailed analysis. Following North et al. (1981) the PDE system investigated in this paper can be summarized as:

$C \frac{\partial T(x, t)}{\partial t}=Q_{0} S(x)\left(\alpha\left(x, x_{s}\right)\right)-(A+B T(x, t))+\frac{\partial}{\partial x} D\left(1-x^{2}\right) \frac{\partial T(x, t)}{\partial x}$

where the terms are defined shortly.

In general, Eq. (1) allows for the computation of the zonally-averaged surface temperature $T$, as function of the time $t$ and the sine of latitude $x=\sin (\phi)$. The model allows for a range of forcing terms, therefore the presence of $\mathrm{CO}_{2}$ forcing and a control function representing the deployment of SRM strategies will be included later. The solution is constrained by boundary conditions, since the heat flux must vanish at the poles and only solutions with no heat transport across the equator are considered.

Increasing the forcing in one hemisphere relative to the other causes a shift of the latitude of zero heat-flux inducing large precipitation anomalies. However, this effect is not taken into account in order to keep the analysis manageable.

The boundary conditions are Neumann boundary conditions and therefore can be expressed as follows North (1975): 
$\frac{\partial T(x, t)}{\partial x}=0 \quad$ with $\quad x=0,1,-1$

where $x=0$ represents the equator, $x=1$ the North Pole and $x=-1$ the South Pole.

Equation (1) represents an EBM where the incoming and outgoing energy are balanced and an equilibrium temperature distribution with latitude is reached. Specifically, $T(x, t)$ represents the annual zonally-averaged temperature field. The incoming energy is the solar radiation, and the energy losses are given by the effect of the Earth's albedo and the infra-red radiation leaving the top of a latitudinal element. The energy transported by a latitudinal element to its neighbours due to the movement of geophysical fluids is represented by a diffusion process where the transport is proportional to the gradient of the temperature field. As is shown later, some of these constants are taken from data in the literature (North et al. 1981; McGuffie and HendersonSellers 2005; Budyko 1969) and others are chosen in order to match the time-domain step response of high-fidelity numerical models. In this way it is possible to regulate the equilibrium climate sensitivity of the system which is a key parameter for comparison between climate models. Therefore, the goal of this model is emulating the behaviour of complex numerical models with a more convenient analytical structure to easily (and rapidly) implement climate engineering control strategies based on SRM.

The problem is split in two separate processes for the two hemispheres, in particular the solution is found for the northern hemisphere when $0<x<1$ and for the southern hemisphere when $-1<x<0$. This operation allows for a more specific description of each hemisphere through the use of appropriate constants. As in North et al. (1981), the outgoing infra-red radiation is well approximated by the expression $I_{I R}=A+B T(x, t)$ Budyko (1969), where $A$ and $B$ are empirical constants selected to account for the effect of clouds, water vapour and $\mathrm{CO}_{2}$. In particular, in Budyko (1969) an infra-red parametrization for the northern and southern hemispheres is found and $I_{I R}$ can be written as:

$I_{I R}=A+B T(x, t)=A_{1}+A_{2} A_{c}+\left(B_{1}+B_{2} A_{c}\right) T(x, t)$

where $A_{1}=257 \mathrm{~W} / \mathrm{m}^{2}, \quad A_{2}=-91 \mathrm{~W} / \mathrm{m}^{2}, \quad B_{1}=1.63$ $\mathrm{W} / \mathrm{m}^{2} /{ }^{\circ} \mathrm{C}, \quad B_{2}=-0.11 \mathrm{~W} / \mathrm{m}^{2} /{ }^{\circ} \mathrm{C}$ for the northern hemisphere and $A_{1}=262 \mathrm{~W} / \mathrm{m}^{2}, A_{2}=-81 \mathrm{~W} / \mathrm{m}^{2}$, $B_{1}=1.64 \mathrm{~W} / \mathrm{m}^{2} /{ }^{\circ} \mathrm{C}, B_{2}=-0.09 \mathrm{~W} / \mathrm{m}^{2} /{ }^{\circ} \mathrm{C}$ for the southern hemisphere, whereas $A_{c}$ is the cloud cover set to 0.5 . Through climatological records of zonal surface temperature and satellite observations this fit has been proven to be quite accurate Cess (1976).

Moreover, $Q_{0}$ is the solar constant given by $342 \mathrm{~W} / \mathrm{m}^{2}$ and $S(x)$ describes the distribution of the incident solar radiation averaged over 1 year for which the expression used in North (1975) is considered: $S(x)=1+S_{2} P_{2}(x)$ where
$S_{2}=-0.477$ is a constant and $P_{2}(x)=\frac{1}{2}\left(3 x^{2}-1\right)$ is the second Legendre polynomial. Then, $\alpha\left(x, x_{s}\right)$ is the planetary coalbedo at latitude $x$ North et al. (1981) for which a smooth albedo formulation, that includes the definition of the ice line at $x=x_{s}$, is considered Widiasih (2013):

$\alpha\left(x, x_{s}\right)=\frac{\alpha_{w}+\alpha_{i}}{2}+\frac{\alpha_{i}-\alpha_{w}}{2} \tanh \left(M\left(x-x_{s}\right)\right)$

where $\alpha_{w}$ is the water co-albedo set to $1-0.32, \alpha_{i}$ is the ice co-albedo set to $1-0.55$ and the parameter $M$ represents the steepness of the albedo function near the ice line and is set to 12 . The value of $x_{s}$ for the current climate is set to $x_{s 0}= \pm 0.95$ for the northern and southern hemisphere, respectively. Moreover, in Eq. (1) $D$ is an empirical constant describing the latitudinal transport of energy. Its value for the northern hemisphere is given by $0.649 \mathrm{~W} / \mathrm{m}^{2} /{ }^{\circ} \mathrm{C}$ North et al. (1981), whereas the value for the southern hemisphere is found in order to satisfy the condition $T_{N}(0, \infty)=T_{S}(0, \infty)$, where $T_{N / S}(x, \infty)$ are the temperature fields at latitude $x$ for the northern and southern hemispheres in the equilibrium state $(t \rightarrow \infty)$. The value of $D$ for the southern hemisphere that satisfies this condition is $0.73 \mathrm{~W} / \mathrm{m}^{2} /{ }^{\circ} \mathrm{C}$.

Finally, in Eq. (1) $C$ is the effective heat capacity, which is largely determined by the oceans. The values of the heat capacities are estimated for the northern and southern hemispheres considering the different hemispherical distributions of land and water. In particular, a larger fraction of water can be found in the southern hemisphere and this leads to a larger heat capacity. The heat capacity over land is approximately $1 / 30$ of the capacity over the ocean mixed layer North et al. (1981). Therefore considering the fraction of water and land in each hemisphere (oceans cover the $61 \%$ of the northern hemisphere and the $82 \%$ of the southern hemisphere) the heat capacity, in terms of $B$, is $2.88 B$ years for the northern hemisphere and $3.79 B$ years for the southern hemisphere.

It is worth noting that the model neglects the mean circulation in both the atmosphere and oceans but includes heat transport due to circulation. This approach allows for tractable mathematics and analytical solutions.

The system in Eq. (1) with the boundary conditions in Eq. (2) can be identified as the non-homogeneous heat equation with Neumann boundary conditions and has an analytical solution. An efficient, straightforward approach for solving such problems and obtaining an analytical solution is provided by the Green's function formalism. Green's functions are constructed by utilizing the eigenfunctions and eigenvalues of the differential operators from which the system is constructed Cole et al. (2010). Once the Green's function for a given problem is known, the solution for the latitudinal distribution of the temperature is immediately computed 
from the analytical expression for the Green's function Hahn and Ozisik (2012).

The general solution in terms of the Green's function can be written as Cole et al. (2010):

$$
\begin{aligned}
T(x, t)= & \int_{\xi_{0}}^{\xi_{f}} T(\xi, 0) G(x, \xi, t, 0) d \xi \\
& +\int_{0}^{t} \int_{\xi_{0}}^{\xi_{f}} G(x, \xi, t, \tau) \Phi(\xi, \tau) d \xi d \tau
\end{aligned}
$$

where $G(x, \xi, t, \tau)$ is the Green's function associated with the differential operators in Eq. (1) with Neumann boundary conditions and can be written as Cole et al. (2010):

$$
\begin{aligned}
G(x, \xi, t, \tau) & =G_{x}(x, \xi) G_{t}(t, \tau) \\
& =\sum_{n=1}^{\infty}(2 n+1) \frac{P(n, x) P(n, \xi)}{L_{n}} \frac{1}{C} \exp \left(-\frac{t-\tau}{L_{t}}\right)
\end{aligned}
$$

where $L_{n}=(n(n+1) D+B)$ are the eigenvalues of the linear operator in the energy balance equation and $L_{t}$ is the time constant given approximately by $C \beta_{0} / B$, where $\beta_{0}$ is the climate sensitivity of the model as determined later. Also, $P(n, x)$ is the nth-degree Legendre Polynomial computed in $x$. The Green's function $G(x, \xi, t, \tau)$ represents the temperature perturbation $\delta T(x, t)$, at latitude $x$, at time $t$, due to an instantaneous heat source of unit strength, located at $\xi$, releasing its energy instantaneously at time $\tau$. Therefore, the argument ' $\xi, \tau$ ' in Eq. (6) represents the impulse, given by the heat source term Hahn and Ozisik (2012), whereas ' $x$, $t$ ' represent the resulting effect. Moreover, $\Phi$ is the external forcing of the system which in this case depends only on $x$ and can be written as:

$\Phi(x)=Q_{0} S(x)\left(\alpha\left(x, x_{s}\right)\right)-A$

In general, the first term on the right-hand side of Eq. (5) is the contribution of the initial condition given by the Green's function, evaluated at $\tau=0$, multiplied by $T(x, 0)$ and integrated over the hemisphere. Whereas, the second term represents the contribution of the forcing to the temperature profile. Then $T(x, 0)$ is the latitudinal distribution of the temperature (at $t=0$ ) which can be found through a similar procedure considering the 1D-model. Its analytical expression is given by:

$T(x, 0)=\int_{\xi_{0}}^{\xi_{f}} G_{x}(x, \xi) \Phi(\xi) d \xi$

where $G_{x}(x, \xi)$ is the part of the Green's function in Eq. (6) that is associated with the differential operator $-D \frac{d}{d x}\left(1-x^{2}\right) \frac{d}{d x}+B$, i.e. the time-independent part. The annual zonally-averaged surface equilibrium temperature for the two hemispheres can be obtained considering $T(x, t)$ in Eq. (5) at the final time $t=t_{f}$. The result is reported in
Fig. 1 and is consistent with the literature (North et al. 1981; Budyko 1969; Schneider and Gal-Chen 1973). Considering the combination of the responses in the two hemispheres, the step response of the PDE model for the climate system can be found in Fig. 2 together with the response from a first order linear model (dashed line) Kravitz et al. (2016) and a semi-infinite diffusion model MacMynowski et al. (2011). The behaviour of the PDE model is comparable with the semi-infinite diffusion model. In particular, the two curves (continuous black thick and thin lines) reach the same equilibrium value, although the relaxation profile is different. In the semi-infinite diffusion model, the overall heat capacity is given by 4.063 years with an equilibrium climate sensitivity of $2.71^{\circ} \mathrm{C}$ MacMynowski et al. (2011), which is comparable with the HadCM3L model. As can be seen in Fig. 2, in this case, the perturbation is considered with respect to the equilibrium temperature. This version of the model, where only the temperature anomaly with respect to the equilibrium

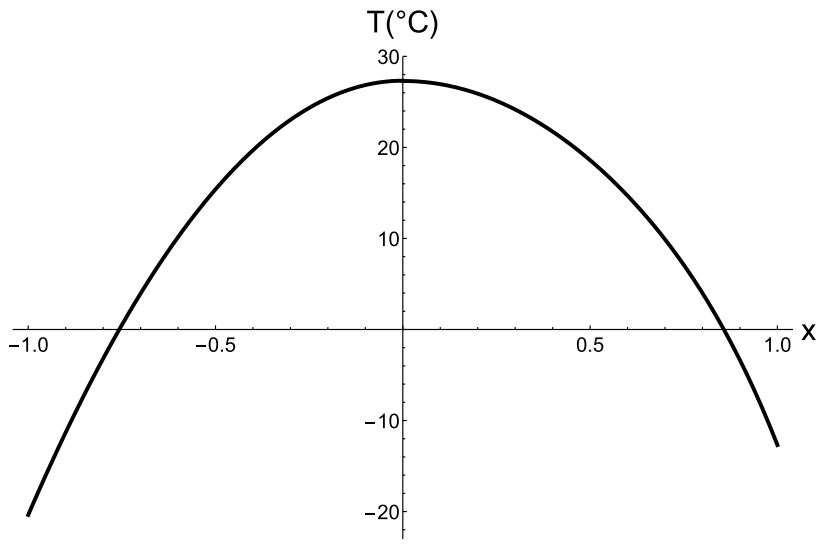

Fig. 1 Latitudinal distribution of the annual zonally-averaged surface equilibrium temperature for the northern and southern hemispheres

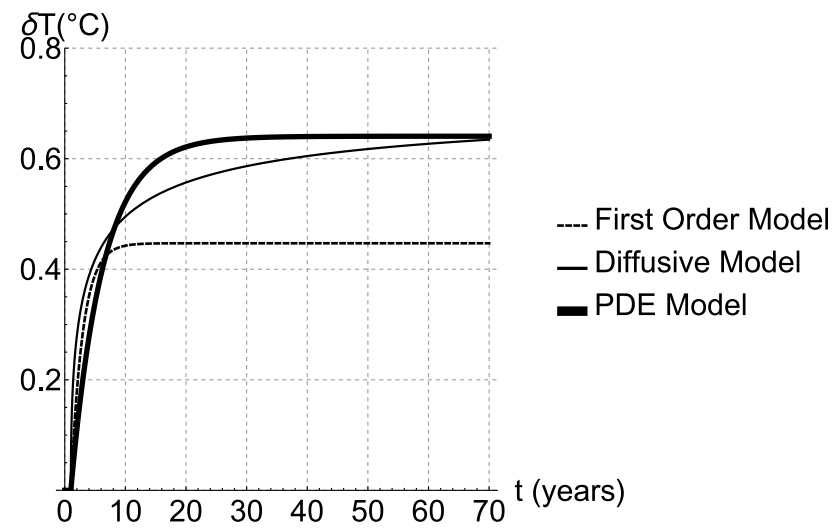

Fig. 2 Step response for the global mean temperature in the time domain due to the step change in radiative forcing of $1 \mathrm{~W} / \mathrm{m}^{2}$ at $t=1$ year of the climate model reported in Eq. (1) (black thick solid line), a first order linear model (dashed line) Kravitz et al. (2016) and a semi-infinite diffusion model (black thin solid line) Kravitz et al. (2016), MacMynowski et al. (2011) 
state is considered, is described in detail in the next section. According to Robert and North (1979) and North et al. (1981), the fundamental sensitivity of the system can be estimated for the PDE model as follows:

$\beta_{0}=\frac{Q_{0}}{100}\left(\int_{0}^{1} \int_{0}^{1} G_{x}(x, \xi) S(\xi)\left(\alpha\left(\xi, x_{s 0}\right)+Q_{0} \frac{d x_{s}}{d Q} \frac{\partial \alpha\left(\xi, x_{s}\right)}{\partial x_{s}}\right) d \xi d x\right)$

where $d x_{s} / d Q$ is the slope surface of the ice line $\left(x_{s}\right)$, i.e. the latitude where the temperature is $-10^{\circ} \mathrm{C}$ (ice line) and the surface is assumed to be covered by ice. In the current climate the ice line is at $x_{s 0}= \pm 0.95$. The value for the slope of the ice line can be found considering Eq. (2.9) in North et al. (1981). The term $\partial \alpha\left(x, x_{s}\right) / \partial x_{s}$ is the derivative of the co-albedo function in Eq. (4) with respect to the ice line calculated at $x_{s 0}$. Computing Eq. (9) for the southern and northern hemisphere, it can be demonstrated that the averaged sensitivity of the PDE model in Eq. (1) with the chosen parameters is $2.32^{\circ} \mathrm{C}$.

\section{Multi-objective control strategies}

It is important to highlight that the expression for the external forcing $\Phi(x, t)$ is a generic function; therefore, it can be used to implement climate engineering strategies and analyse the behaviour of the PDE model. In this section a slightly modified version of the model in Eq. (1), where the system is considered in the neighbourhood of its equilibrium state, is presented.

This version includes external forcing which represents the excess of carbon dioxide in the atmosphere. This forcing term $\left(F_{\mathrm{CO}_{2}}\right)$ causes an imbalance in the radiative forcing in Eq. (1) producing an anomaly in the latitudinal temperature profile. In this model, the anomaly indicates exclusively the effect of an excess of $\mathrm{CO}_{2}$ in the atmosphere. In particular, the $1 \mathrm{pctCO}_{2}$ scenario Collins et al. (2013), where a constant increase in carbon dioxide concentration of $1 \%$ per year is assumed, is considered in this section.

Assuming the deployment of a climate engineering strategy consisting of a reduction of the incoming solar radiation (SRM), a control function, defined as $U(x, t) Q_{0} S(x) \alpha\left(x, x_{s}\right)$, which in general is a function of both latitude and time, will represent the climate engineering intervention in terms of the fractional reduction of the incoming solar radiation. This term is included in the model through Eqs. (10-11) and aims to reduce the temperature anomaly generated by the excess of atmospheric $\mathrm{CO}_{2}$.

Thus, considering a small radiative perturbation $\delta F_{\mathrm{CO}_{2}}$, it is possible to solve the equation governing the temperature perturbation as follow:

$\delta T(x, t)=\int_{\xi_{0}}^{\xi_{f}} \int_{0}^{t} G(x, \xi, t, \tau) \Phi(x, t) d \tau d \xi$ where, in this case, $\Phi$ is given by the forcing due to atmospheric $\mathrm{CO}_{2}$ and the deployment of climate engineering intervention:

$\Phi(x, t)=\delta F_{\mathrm{CO}_{2}}(x, t)-U(x, t) Q_{0} S(x) \alpha\left(x, x_{s}\right)$

As can be noted, since only perturbations around the equilibrium temperature of the system are considered, the term depending on $T(x, 0)$ is equal to zero and only the perturbative term of Eq. (5) is considered in Eq. (10).

The analytical expression for the temperature distribution, obtained through the Green's function approach, allows for a fast and efficient investigation of the effects of SRM deployment on the climate system. The advantages of using such an approximate mathematical model for closed-loop control purposes can be summarised as follow: (1) capturing latitudinal disparities in induced cooling; (2) easy application of optimization processes and multi-objective analyses; (3) clearer understanding of the key climatic processes involved and the effects of closed-loop control on them; (4) the possibility of developing an analytical control function with latitudinal resolution; (5) the efficient assessment of new climate engineering strategies, prior to more detailed analysis using GCMs.

The 1 pctCO $\mathrm{O}_{2}$ scenario assumes that the $\mathrm{CO}_{2}$ concentration in the atmosphere rises steadily at $1 \%$ per year; therefore, the associated radiative forcing can be obtained through the following relationship:

$F_{\mathrm{CO}_{2}}(t)=5.35 \log \left(\frac{c c}{c c_{0}} e^{\lambda t}\right) \quad\left(\mathrm{W} / \mathrm{m}^{2}\right)$

where the expression found in Gasser et al. (2015) for the radiative forcing due to atmospheric $\mathrm{CO}_{2}$ is considered. Here, $c c$ is the current $\mathrm{CO}_{2}$ concentration in the atmosphere [400 ppm Dlugokencky and Tans (2016)], $c c_{0}$ is the preindustrial level of carbon dioxide [278 ppm Dlugokencky and Tans (2016)] and $\lambda=\log (1.01)$ represents the $1 \%$ per year growth rate.

The model described offers an analytical approach to the design of control strategies to counteract radiative forcing on a latitudinal basis. Following the same approach used in Kravitz et al. (2016), a multi-objective control strategy is applied to the system. The objectives regard the minimization of changes in the global mean temperature, the temperature gradient and the equator-to-pole temperature gradient due to increasing $\mathrm{CO}_{2}$. For this purpose the functions to minimize are defined by the projection of the temperature distribution $\delta T(x, t)$ onto the first three Legendre polynomial functions Ban-Weiss andCaldeira (2010); Kravitz et al. (2016) that are reported below:

$L_{0}=1$

$L_{1}(x)=x$ 


$$
L_{2}(x)=\frac{1}{2}\left(3 x^{2}-1\right)
$$

Considering Eqs. (13-15) and the analytical solution of the PDE system for the temperature anomaly in Eq. (10), the three outputs can be defined as follow:

$\delta T_{i}(t)=\int_{x_{0}}^{x_{f}} \delta T(x, t) L_{i}(x) d x \quad i=0,1,2$

with $x_{0}=\sin \left(\phi_{0}\right)$ and $x_{f}=\sin \left(\phi_{f}\right)$, where subscripts 0 and $f$ represent the extremes of the hemispheric integration.

As in Kravitz et al. (2016), the first goal will now consider the minimization of the global mean temperature $\delta T_{0}(t)$ only (case 1), the second case also considers the minimization of the temperature gradient $\delta T_{1}(t)$ (case 2), and the third case investigates the full problem where all the three objectives are taken into account (case 3).

A Proportional-Integral (PI) control is employed to achieve the required control objectives. This control structure is a feedback control strategy, where the extent of the control at time $t$ depends on the state $\delta T_{i}$ at the previous time $(t-1)$ (years). This approach is justified considering a reasonable time delay for the deployment of SRM strategies. The observations of the temperature distribution during year $t$ would be used to estimate the quantity of material required, for example if stratospheric aerosol injection is used. The time to collect data, implement decision-making processes are assumed to cause a delay between the time of observation and the deployment of the climate engineering strategy. In this paper a time-delay of 1 year is considered. Other important properties of feedback control systems and advantages of their use in climate engineering can be found in MacMartin et al. (2014) and MacMartin et al. (2014).

The general structure of the PI control used to achieve the objectives is defined as:

$U_{i}(t)=k_{P i} \delta T_{i}(t-1)+k_{I i} \int_{0}^{t} \delta T_{i}(t-1) d t \quad i=0,1,2$

where $U_{0}(t)$ aims to minimize the global mean temperature $\delta T_{0}(t), U_{1}(t)$ regards the control of $\delta T_{1}(t)$ and $U_{2}(t)$ of $\delta T_{2}(t)$, respectively. The control functions for cases (1), (2) and (3) can be written as:

$U_{1 x 1}(t)=U_{0}(t) L_{0}$

$U_{2 \times 2}(x, t)=U_{0}(t) L_{0}+U_{1}(t) L_{1}(x)$

$U_{3 \times 3}(x, t)=U_{0}(t) L_{0}+U_{1}(t) L_{1}(x)+U_{2}(t) L_{2}(x)$

As can be seen from Eqs. (18-20), the control functions are given by a combination of the functions $U_{i}(t)$, describing the time-history of the control (given by the outcomes of the PI control strategy) and the functions $L_{i}(x)$, characterizing the latitudinal distribution of the control.

\subsection{Results}

Considering the analytical solution of the PDE system in Eq. (10), the three outputs, $\delta T_{0}(t), \delta T_{1}(t)$ and $\delta T_{2}(t)$, can be computed through Eq. (16). As noted earlier, climate engineering through the reduction of incoming solar radiation is deployed in case (1) to minimize the global mean temperature, in case (2) to drive both $\delta T_{0}(t)$ and the temperature gradient $\delta T_{1}(t)$ to zero and, finally, in case (3), all three outputs are controlled. Again, in this analysis the $1 \mathrm{pctCO}_{2}$ scenario is considered. To achieve these strategies, the control functions reported in Eqs. (18-20) are employed.

The three control strategies are summarized in Table 1. Specifically, the controlled outputs are indicated with the symbol " $\star$ " in the first three columns, whereas, in the last column the control function employed is reported for each case. Values for $k_{P i}$ and $k_{I i}(\mathrm{i}=1,2,3)$ are chosen in order to ensure a fast response whilst avoiding excessively increasing the system's sensitivity to natural variability MacMartin et al. (2014). In particular, a convergence time of 3 years is assumed to select the gains for $U_{0}, U_{1}$ and $U_{2}$ in Eq. (17).

The PI-control scheme is now fully defined and the control functions, in terms of the reduction of insolation, obtained. Figure 3 a shows the time-history of $U_{1 x 1}(t)$, which has a uniform distribution at every latitude since the first Legendre polynomial $L_{0}$ does not depend on $x$. This strategy shows the effect of a latitudinally-uniform reduction of insolation that increases with time. However, although the increase of atmospheric $\mathrm{CO}_{2}$ is uniformly distributed there is an amplified effect at the poles. Therefore, when uniform cooling is applied as in case (1), an overcooling of the tropics and an undercooling of the poles occurs with the northern hemisphere cooler than the southern hemisphere [see also Kravitz et al. (2016)]. This result can be seen from Fig. 5 where the latitudinal distribution of the zonal mean temperature at the final time is reported for the three cases.

Hemispheric differences are related to the different distribution of ocean and land between the two hemispheres and, in particular, to the impact of the ocean on heat transport Kang and Seager (2012). These effects are taken into account in the PDE model through the values of the heat

Table 1 Summary of the control strategies considered

\begin{tabular}{lllll}
\hline case & $\delta T_{0}(t)$ & $\delta T_{1}(t)$ & $\delta T_{2}(t)$ & Control function \\
\hline 1 & $\star$ & - & - & $U_{1 x 1}(t)$ \\
2 & $\star$ & $\star$ & - & $U_{2 x 2}(x, t)$ \\
3 & $\star$ & $\star$ & $\star$ & $U_{3 x 3}(x, t)$ \\
\hline
\end{tabular}


a

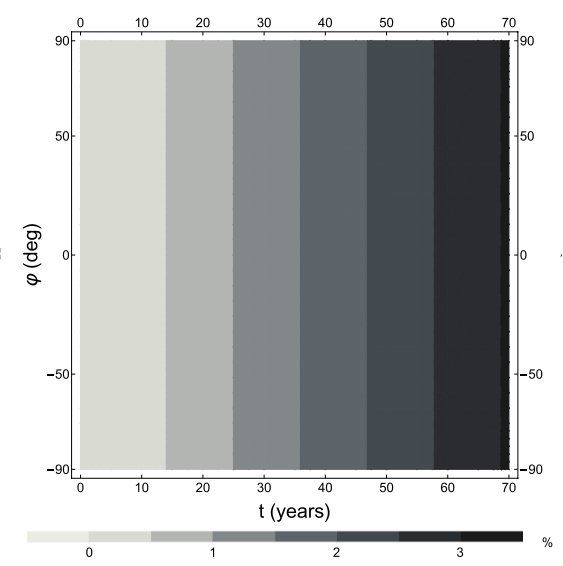

b

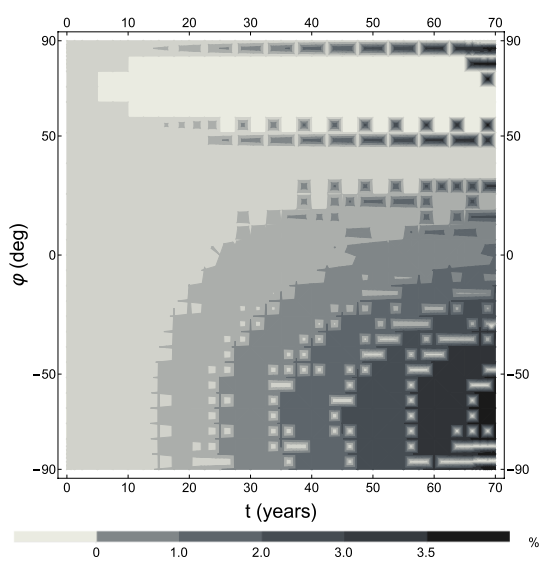

c

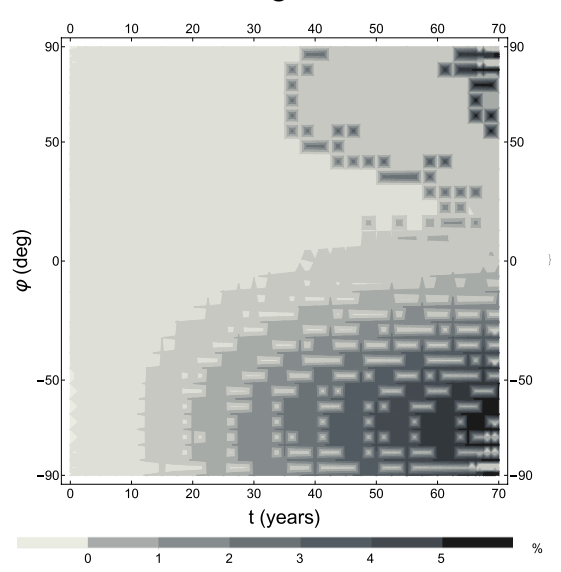

Fig. 3 Latitudinal distribution of the control function $\mathbf{a} U_{1 \times 1}, \mathbf{b} U_{2 \times 2}$ and $\mathbf{c} U_{3 \times 3}$ with the time

capacities and the transport coefficients employed for the northern and southern hemispheres.

Figure $3 \mathrm{~b}$ shows the function $U_{2 \times 2}(x, t)$, employed in the case (2), which is given by the combination of the feedback control of $\delta T_{0}(t)$ and $\delta T_{1}(t)$. Its latitudinal distribution is dictated by the first and the second Legendre polynomial expressions in order to minimize the global mean temperature as well as the temperature gradient.

The additional feedback control of the inter-hemispheric temperature gradient in case (2) reduces disparities between the temperature residuals in the north pole and the south pole and decreases over-cooling of the tropics. Therefore, as can be seen in Fig. 3b, a larger cooling effect is required in the southern hemisphere.

As noted in Kravitz et al. (2016), the additional feedback control of the inter-hemispheric temperature gradient in case (3) reduces disparities between the temperature residuals in the north pole and the south pole and decreases over-cooling of the tropics. Again, these effects are also confirmed in Fig. 5.

Finally, Fig. 3c shows the distribution of the control function employed in the full case (3), where the equator-to-pole temperature gradient is also minimized.

In all three cases the control strategy employed is consistently comparable with the numerical results obtained in Kravitz et al. (2016), where multi-objective control strategies are applied to two fully coupled atmosphere-ocean general circulation models (AOGCM) that participated in CMIP5, the CESM 1.0.2 (Community Earth System Model) and the GISS ModelE2 (the Goddard Institute for Space Studies) in order to minimize $\delta T_{0}(t), \delta T_{1}(t)$ and $\delta T_{2}(t)$. In particular, the control responses obtained with the analytical solution of the PDE model are comparable with those obtained through the CESM. However the model shows low sensitivity to the control of $L_{1}$ as occurs for the GISS model.
The results regarding the three outputs of the multi-objective control strategy simulations are reported in Fig. $4 \mathrm{a}-\mathrm{c}$. In particular, the output time-history of $\delta T_{0}(t)$ is reported in Fig. 4a, whereas Fig. 4b, c show $\delta T_{1}(t)$ and $\delta T_{2}(t)$, respectively. Each figure includes three curves: the black line represents case (1), the dark-grey line represents case (2) and the light-grey line shows the full case (3). Therefore, it is possible to analyse the effect of every control strategy on each output. Moreover, a Gaussian noise (zero mean and standard deviation set to $10^{-2}$ ) is added to the outputs of the temperature level to simulate measurement noise and climate variability.

It can be seen from Fig. 4a-c that, in all the cases considered, the objective of each specific requirement is achieved. In fact the global mean temperature in case (1), the interhemispheric temperature gradient in case (2) and the equator-to-pole temperature gradient in case (3) are minimized. Although, from Fig. 4b, it can be noted that the system is not very sensitive to $U_{1}$ and it is found that $\delta T_{1}$ is reduced to a mean value of approximately $0.02{ }^{\circ} \mathrm{C}$. This result is comparable with the outcome from the GISS model found in Kravitz et al. (2016). Moreover, negative effects are found for the objectives that are not managed in a particular case, such as $\delta T_{1}$ in cases (1) and (3) and $\delta T_{2}$ in cases (1) and (2).

In accordance with results from the literature Kravitz et al. (2016); Kravitz et al. (2011), the required latitudinallyuniform reduction of insolation increases linearly with time as the atmospheric $\mathrm{CO}_{2}$ concentration grows (see Fig. 3a) and mainly aims to decrease the global mean temperature $\delta T_{0}$.

With respect to similar simulations from the literature, the results for $\delta T_{0}(t), \delta T_{1}(t)$ and $\delta T_{2}(t)$ are comparable with results reported in Kravitz et al. (2016).

Finally, in a rather similar way to the literature the equator-to-pole temperature gradient $\left(\delta T_{2}\right)$ in Fig. $4 \mathrm{c}$ shows 


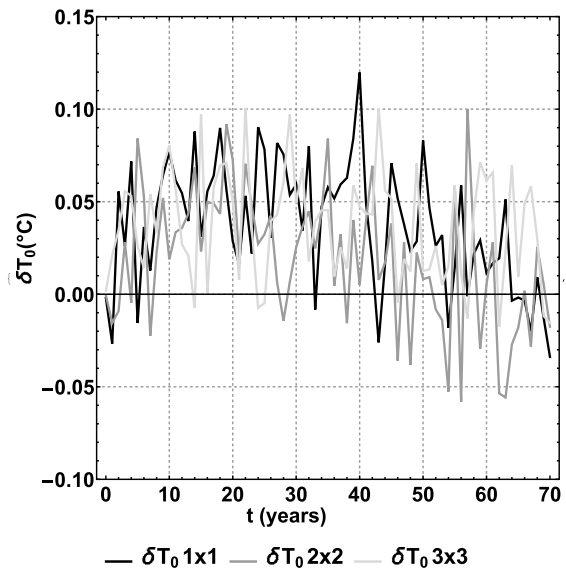

b

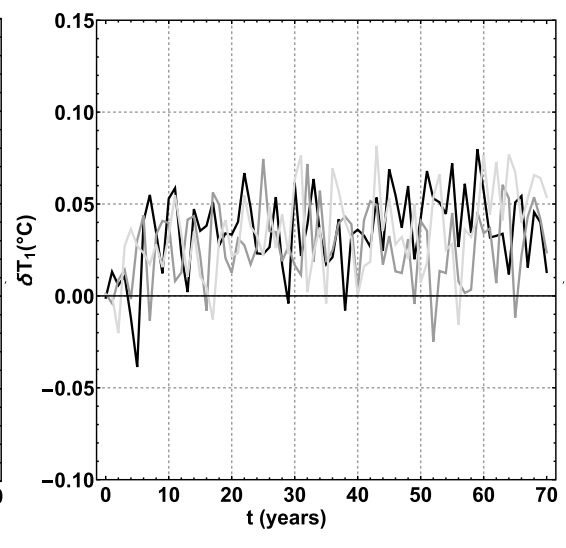

$-\delta \mathrm{T}_{1} 1 \times 1-\delta \mathrm{T}_{1} 2 \times 2-\delta \mathrm{T}_{1} 3 \times 3$
C

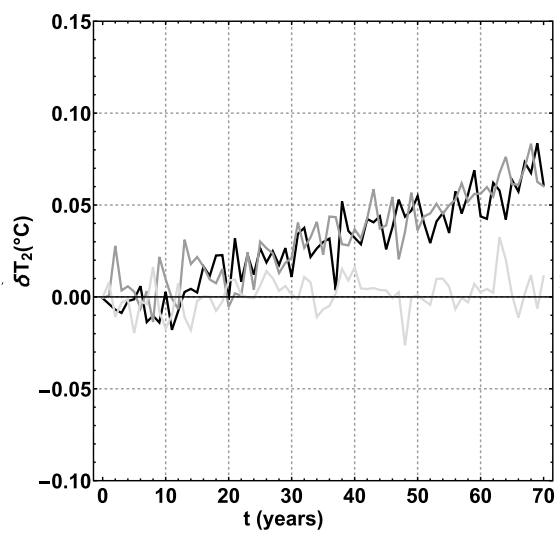

$-\delta \mathrm{T}_{2} 1 \times 1-\delta \mathrm{T}_{2} 2 \times 2-\delta \mathrm{T}_{2} 3 \times 3$

Fig. 4 Perturbation of the global mean temperature $\delta T_{0}\left({ }^{\circ} \mathrm{C}\right)(\mathbf{a})$, the inter-hemispheric temperature gradient $\delta T_{1}\left({ }^{\circ} \mathrm{C}\right)(\mathbf{b})$ and the equator-to-pole temperature gradient $\delta T_{2}\left({ }^{\circ} \mathrm{C}\right)$ (c) in cases 1 (black line), 2 (dark-grey line), 3 (light-grey line)

Fig. 5 Latitudinal distribution of the zonal mean temperature at final time $\left(t_{f}=70\right.$ years) for the three considered cases

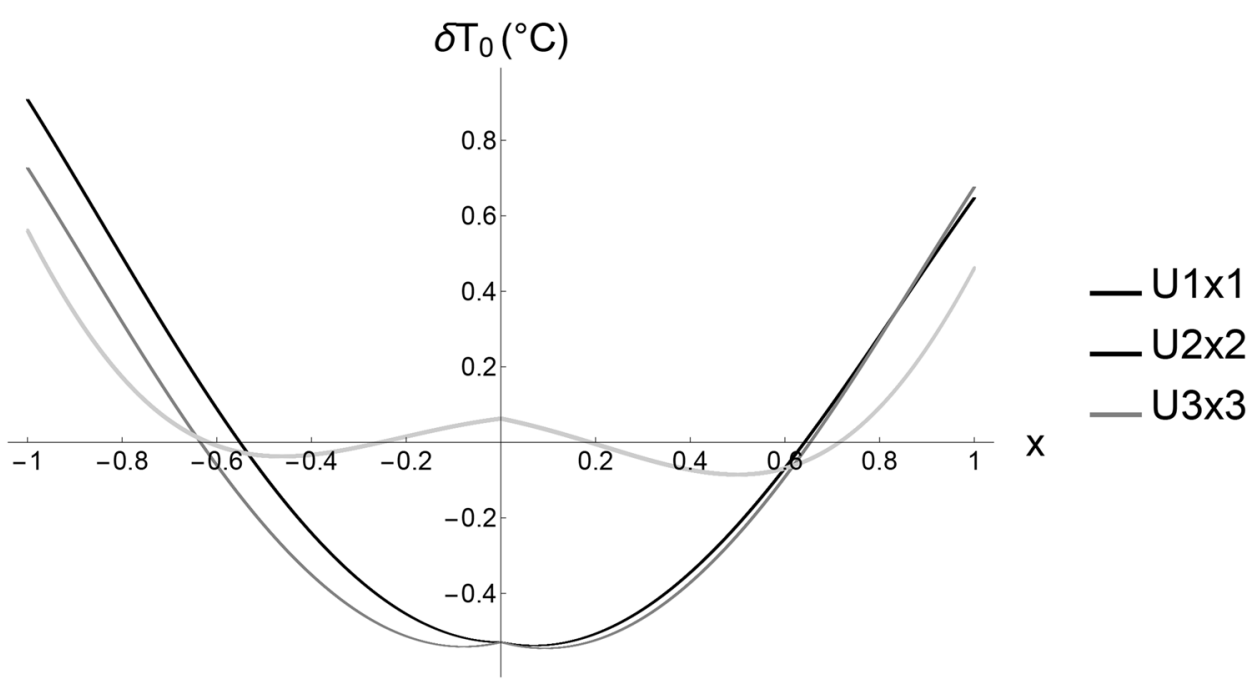

convergence to zero steady-state error in case (3), while in case (1) shows large sensitivity to the climate variability (noise). It is therefore clear that the analytic PDE model can provide an efficient and effective means of investigating non-uniform climate engineering strategies.

Moreover, the model described in this paper allows also for the analysis of the zonal mean temperature. Therefore, a trade-off between the number of controlled degrees of freedom of SRM and the compensation of the zonal mean temperature is performed. Considering Eq. (10), the zonal mean temperature anomaly is computed and it is found that the rms error in compensating $\delta T$ is $0.84{ }^{\circ} \mathrm{C}, 0.81^{\circ} \mathrm{C}$ and $0.31^{\circ} \mathrm{C}$ when $U_{1 \times 1}, U_{2 \times 2}$ and $U_{3 \times 3}$ are applied to the system respectively. These results are shown in Fig. 5, where the zonal mean temperature at final time is reported with latitude for the three cases considered. As can be seen, the temperature anomaly is noticeably lower in case (3). This result demonstrates that the zonal mean temperature is not completely minimized in any case, but that the overall rms error decreases when more degrees of freedom are managed. In fact, in the case when SRM is not deployed $(U=0)$ the overall rms error due to the $1 \mathrm{pctCO}_{2}$ scenario is $1.41^{\circ} \mathrm{C}$. Thus, the computed control functions are able to manage the reduction of the global mean temperature anomaly, the temperature gradient and equator-to-pole temperature gradient, and greater benefits are found for the zonal mean temperature in all the three cases. In particular, case 3 is the most advantageous and indicates larger residuals of the zonal mean temperature anomaly when 3 degrees of freedom are considered.

This outcome is also confirmed in Ban-Weiss and Caldeira (2010), where several combinations of $L_{0}, L_{1}$ and $L_{2}$ distributions are employed for SRM. In particular, it is found that the rms zonal mean land temperature change from a doubling of 
$\mathrm{CO}_{2}$ is reduced when more degrees of freedom are considered. Also, it is found that with a uniform SRM distribution and increased control closer to the poles, there is a more nearly uniform offsetting of $\mathrm{CO}_{2}$-induced warming that restores global mean temperature without over-cooling the equatorial regions and under-cooling the polar regions. This can also be demonstrated through the PDE model when the $1 \mathrm{pctCO}_{2}$ scenario is considered. As already noted in Sect. 4, the PDE model can be employed to find a control law with latitudinal resolution to obtain a desired temperature profile. Therefore, the analytical solution reported in Eq. (5) is used to reduce to zero the rms zonal mean temperature and so Fig. 6 is obtained. In particular, the control law has been found by setting the expression of the rms zonal mean temperature provided through the PDE model to zero. As expected, the radiative forcing required to counteract the $1 \mathrm{pctCO} \mathrm{O}_{2}$ scenario increases with time (see left panel in Fig. 6) and is larger at the poles than the equator in order to avoid the under-cooling that would be caused by a uniform deployment of SRM. In particular, this can be noted from the right panel of Fig. 6, where the latitudinal distribution of the control law at the final time of the simulation (when the radiative forcing is at its maximum) is reported. This method is equivalent to the selection of the necessary combination of $L_{0}, L_{1}$ and $L_{2}$. In particular, it is found that small levels of control over $L_{1}$ are required. As can be seen, the PDE model quickly produces the control function required to achieve the required goals.

Other quantitative analysis of PI, or other control laws, can again be efficiently performed using the PDE model.

\section{Design of a control law to counteract a doubling of $\mathrm{CO}_{2}$}

The analytical solution of the PDE model reported in Eq. (5) can be employed to find a control law with latitudinal resolution to obtain a desired temperature profile. As demonstrated in the previous section, the PI-control is used in a closed-loop scheme to achieve the minimization of the required objectives. The final outcome is the appropriate time-dependent control function that takes into account latitudinal disparities.

In this section, a more generic case is investigated. The method required to find a control function to achieve a specific temperature profile is presented. Assuming a doubling of $\mathrm{CO}_{2}$ (which amounts to a forcing of $F_{2 \times \mathrm{CO}_{2}}=3.71 \mathrm{~W} / \mathrm{m}^{2}$ ) the necessary reduction of solar insolation to drive the temperature to the pre-industrial profile is evaluated and expressed through a control law with latitudinal resolution.

The analytical solution of the system in Eq. (10), i.e. when small variations of temperature around the equilibrium state are assumed, is considered with external forcing that takes into account the doubling of $\mathrm{CO}_{2}$ and the intervention of climate engineering, as given in Eq. (21):

$\Phi(x, t)=F_{2 \times \mathrm{CO}_{2}}-U(x, t) Q_{0} S(x) \alpha\left(x, x_{s}\right)$

Although the radiative forcing $F_{2 \times \mathrm{CO}_{2}}$ is constant with latitude, the climate engineering intervention consists in the fractional reduction of the incoming solar radiation (given by the term $Q_{0} S(x) \alpha\left(x, x_{s}\right)$, see Fig. 7) and therefore depends on its latitudinal distribution. In this context, the control function $U(x, t)$, required to counteract the effect of $F_{2 \times \mathrm{CO}_{2}}$, can now be found by setting $\Phi(x, t)$ to zero.

Unlike to the $1 \%$ linear increase used in Sect. 3 , in this section a doubling of $\mathrm{CO}_{2}$ concentration in the atmosphere is considered. In particular, two cases are considered for this simulation: in the first case a gradual change from the preindustrial level up to a doubling of $\mathrm{CO}_{2}$ occurs over 70 years (with a time constant equal to 34.4 years and $F_{\mathrm{CO}_{2}}=F_{2 \times \mathrm{CO}_{2}}$ when $t=70$ years), whereas, in the second case, the external forcing consists of a constant step change in the radiative forcing equal to $F_{2 \times \mathrm{CO}_{2}}$. The output of the first control strategy is given by the control law in Fig. 8a, where the required fractional reduction of insolation is reported for the northern
Fig. 6 Left panel: control law to minimize the rms zonal mean temperature anomaly due to the $1 \mathrm{pctCO}_{2}$ scenario over 70 years. Right panel: latitudinal distribution of the control law at the final time $t_{f}=70$ years
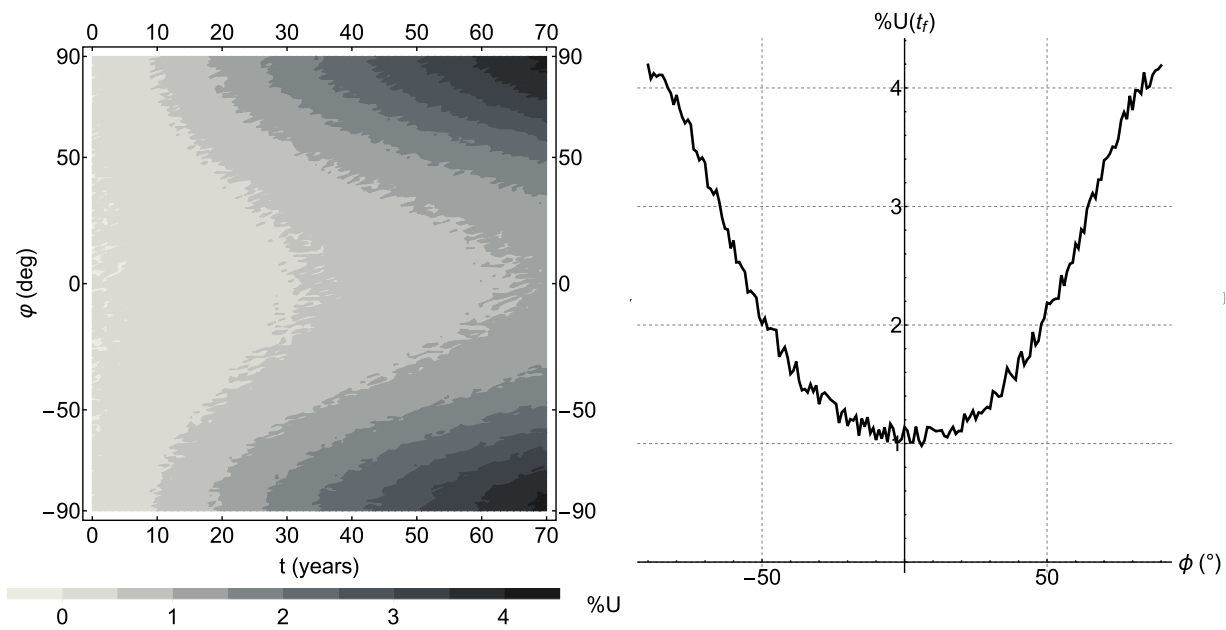


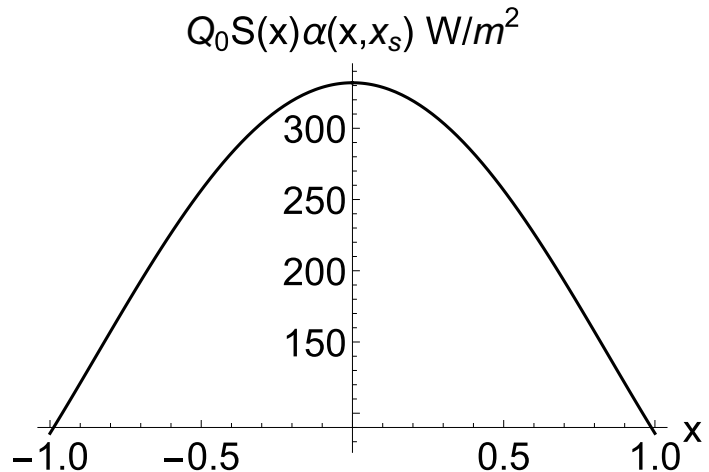

Fig. 7 Incoming solar radiation reaching the Earth's surface as a function of the latitude

and southern hemisphere. As expected, the required control to counteract $F_{\mathrm{CO}_{2}}$ increases with time up to approximately $4 \%$ of the incoming solar radiation at higher latitudes.

In the second case (Fig. 8b), a constant radiative forcing equal to a doubling of $\mathrm{CO}_{2}$ is assumed and a slightly larger control effort is necessary overall. In fact the maximum value of $U(x, t)$ required is $4.5 \%$ of the incoming solar radiation. This is due to the larger temperature anomaly caused by the steady radiative forcing with respect to the gradual change investigated in the first case. Moreover, integrating $U(x, t)$ over latitude, it is possible to estimate the global mean solar insolation required to counteract a doubling of $\mathrm{CO}_{2}$. This is found to be $1.78 \%$, which is comparable with the value of $1.8 \%$ found in literature where the global temperature is investigated for a doubling of the atmospheric $\mathrm{CO}_{2}$ content, such as in Bala et al. (2008) and Govindasamy and Caldeira (2000).

The increase in atmospheric $\mathrm{CO}_{2}$ concentration causes warming everywhere but requires a larger cooling at the poles. This can be justified considering the pattern of the incoming solar radiation (see Fig. 7). When SRM is considered, the latitudinal distribution of the control law is always related to the pattern of insolation and the response of the climate system, which in this paper is given by the PDE model described in Sect. 2.1.

Since the insolation is larger at the equator and lower at the poles, in order for the term $U(x, t) Q_{0} S(x) \alpha\left(x, x_{s}\right)$ to be constant over latitude and balance $F_{2 \times \mathrm{CO}_{2}}$, in both cases investigated, the pattern of $U(x, t)$ is required to have an inverse latitudinal distribution with respect to the incoming solar radiation. Therefore, it is found that the required control is larger at the poles than at the equator. This result is again widely comparable with the literature, for example (Bala et al. 2008; Ban-Weiss andCaldeira 2010; Govindasamy et al. 2003).

\section{Constraints on climate engineering}

In this section the dynamics of the ice line is investigated using the analytical solution of the PDE model in Eq. (1). Climate engineering involves the manipulation of the climate system, therefore the analysis of the stability of the global climate (related to ice line dynamics) is of critical relevance for the study of the impact of climate engineering interventions.

This analysis is of key importance for climate engineering involving SRM since it demonstrates that the extent of the insolation reduction commonly considered for SRM is far from triggering large-scale instability of the climate system. The definition of safe operating boundaries is a requirement for engineering ventures. It will be shown that the PDE can be readily adapted for such analysis.

The Lyapunov stability criterion is now exploited to find the critical climate engineering intervention that would lead the current climate towards an ice-covered state. An upper
Fig. 8 Control law to counteract (a) a time-increasing radiative forcing that reaches $F_{2 \times \mathrm{CO}_{2}}=3.71 \mathrm{~W} / \mathrm{m}^{2}$ in 70 years and $\mathbf{b}$ a constant radiative forcing equal to $F_{2 \times \mathrm{CO}_{2}}$
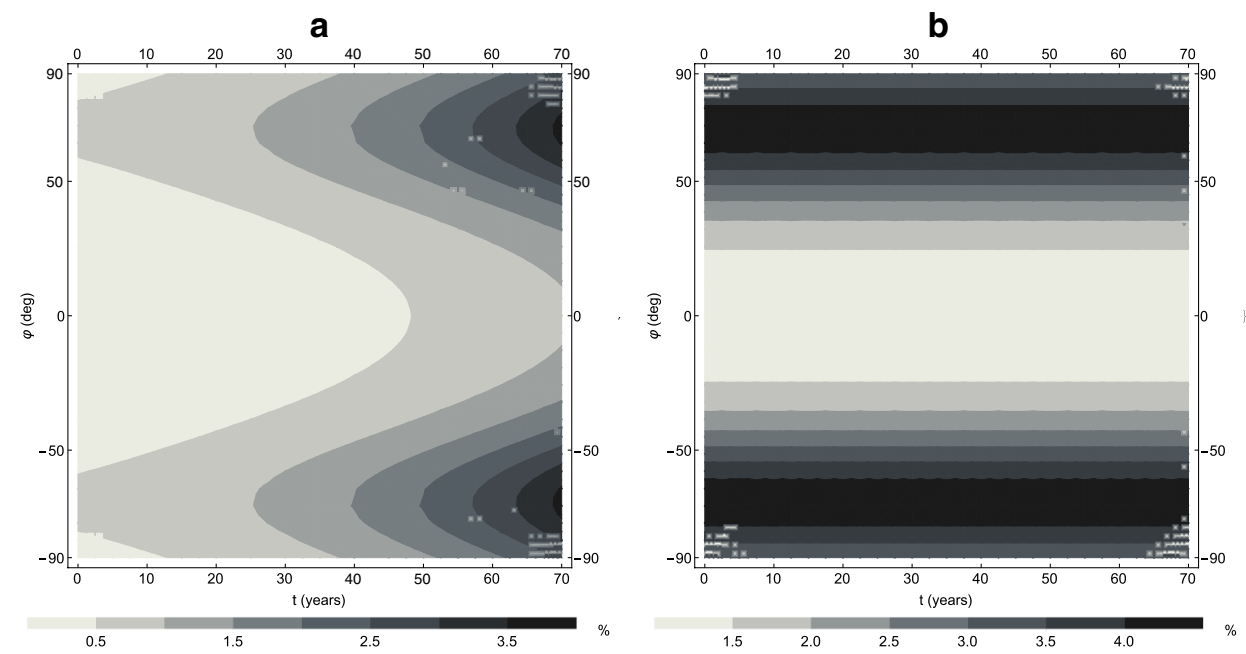
limit on SRM is therefore found by exploring the effect of climate engineering on the dynamics of the ice line. While this limit is of course highly unlikely to be reached, the analytical PDE model provides insight into the extreme operational boundaries of SRM.

For simplicity, this strategy is employed on the northern hemisphere only, therefore $x_{1}=1$ always refers to the North pole and the value employed in this section for the climate sensitivity takes into account only the northern hemisphere $\left(\beta_{0}=2.735^{\circ} \mathrm{C}\right)$. The ice line is defined as the latitude where the temperature reaches $T_{s}=-10^{\circ} \mathrm{C}$ and it is assumed that when the temperature is equal or less than $T_{S}$ the surface is ice-covered and the albedo is 0.55 North et al. (1981). In the current climate the ice line is at $x=x_{s 0}=0.95$. As in North et al. (1981), the temperature at the perturbed ice line is obtained by expanding the ice line condition to first order in small quantities as in Eq. (22):

$T\left(x_{s}+\delta x_{s}, t\right)=T\left(x_{s}, t\right)+\left(\frac{\partial T}{\partial x}\right)_{x_{s}} \delta x_{s}$

where $T\left(x_{s}, t\right)=T\left(x_{s}, \infty\right)+\delta T\left(x_{s}, t\right)$ and $\delta x_{s}$ is the variation of the ice line latitude. Also, $T(x, \infty)$ is the temperature field at latitude $x$ at equilibrium, i.e. for $t \rightarrow \infty$. Because of the ice line condition, Eq. (22) can also be written as $T\left(x_{s}+\delta x_{s}, t\right)=T_{s}=T\left(x_{s}, \infty\right)$, therefore the variation of the ice line latitude is given by Robert and North (1979):

$\delta x_{s}(x, t, U)=\frac{\delta T(x, t, U)}{\left(\frac{\partial T(x, \infty)}{\partial x}\right)_{x_{s}}}$

The numerator of Eq. (23) is the temperature variation at the ice line and can be calculated through the expansion to first order in the small quantities of the time-dependent energy balance equation in Eq. (1). The complete procedure can be found in North et al. (1981), where the analytical expression of $\delta T(x, t, U)$ is found and can be written as:

$\delta T(x, t, U)=e^{-\lambda t / C} \int_{x_{0}}^{x_{1}} G_{Z}(x, \xi) U\left(S(\xi) \alpha\left(\xi, x_{s 0}\right)\right) d \xi$

where $U\left(S(\xi) \alpha\left(\xi, x_{s 0}\right)\right)$ is the perturbation that causes the ice line shift and depends on the control function $U$. For the equator and the pole, respectively, the limits are set as $x_{0}=0$ and $x_{1}=1$. Moreover, $G_{Z}(x, \xi)$ represents the temperature response to a ring of heat added/subtracted at a given latitude and includes the effect of the ice line shift to first order, as found in North et al. (1981).

Moreover, $\lambda$ is the stability eigenvalue of the equilibrium solution $T(x, t)$ under a perturbation $\delta T$. In order to understand the meaning of $\lambda$ it is necessary to report some results from the study of the linear stability of the 1-D model in North et al. (1981). In this study a transcendental equation, which is satisfied only for the stability eigenvalues $\lambda$, is developed and can be written as follow:

$\frac{d Q}{d x_{s}}\left(x_{s 0}\right)=\lambda \frac{Q_{0}^{2}}{A+B T_{s}} \sum_{n} \frac{B S\left(x_{s}\right)\left(P\left(n, x_{s}\right)\right)^{2}}{L_{n}\left(L_{n}-\lambda\right)}=F_{\lambda}$

where $L_{n}, P(n, x), A$ and $B$ are given in Sect. 2.1 and the function $F_{\lambda}$ is given by the right-hand side of Eq. (25). According to North et al. (1981), one way to obtain $d Q / d x_{s}$ is a relationship depending on the climate sensitivity of the model $\left(\beta_{0}\right)$ given by:

$\frac{d Q}{d x_{s}}\left(x_{s}\right)=\frac{Q_{0}^{2} S\left(x_{s}\right) \Delta a}{100 B \beta_{0}-\left(A+B T_{\infty}\right)}$

where, $\Delta a$ is the change of albedo at the ice line equal to $\left|a_{i}-a_{w}\right|=0.23$, and where $a_{i}$ and $a_{w}$ are defined in Sect. 2.1. The functions $\frac{d Q}{d x_{s}}\left(x_{s 0}\right)$ and $F_{\lambda}$ can be plotted as a function of $\lambda$ as shown in Fig. 9. The intersections of the curves provide the roots $\lambda$ of Eq. (25). In the case when $\frac{d Q}{d x_{s}}\left(x_{s 0}\right)<0$ the lowest root is negative and the solution is unstable. This condition represents state II and is equivalent to the transition between state I given by the present climate and state III representing the ice-covered Earth, according to the notation in North et al. (1981).

The three equilibrium states are shown in Fig. 10, where the potential function $\Psi$ normalized over $Q_{0}$ is illustrated as a function of $T_{0}$. Here, $T_{0}$ is the global equilibrium temperature, which is defined as the integral of $T(x, 0)$ (see Eq. 8) with respect to latitude.

The expression for $\Psi\left(T_{0}\right)$ can be found in North et al. (1981) and it is given by:

$\Psi\left(T_{0}\right)=A T_{0}+\frac{1}{2} B T_{0}^{2}-Q_{0} \int_{0}^{T_{0}} H_{0}\left(T_{0}^{\prime}\right) d T_{0}^{\prime}$

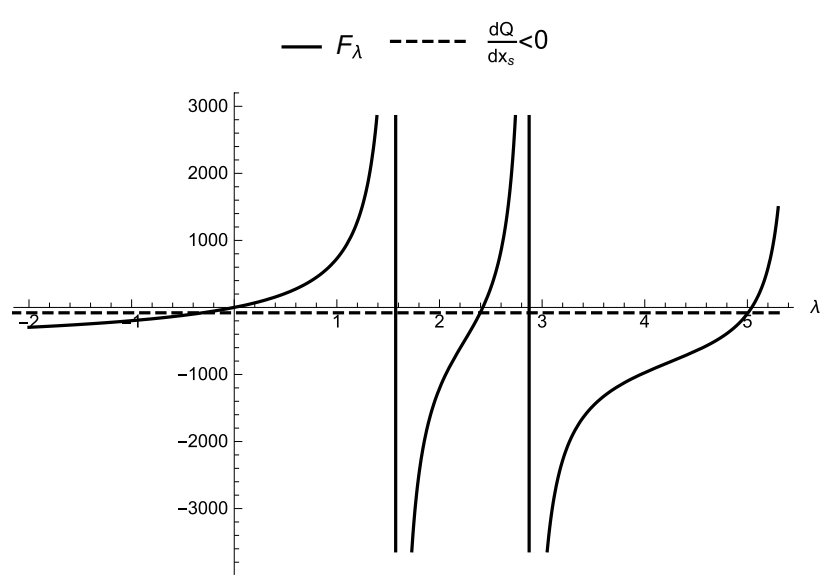

Fig. 9 Plot of the functions $\frac{d Q}{d x_{s}}$ and $F_{\lambda}$ versus the stability parameter $\lambda$ 


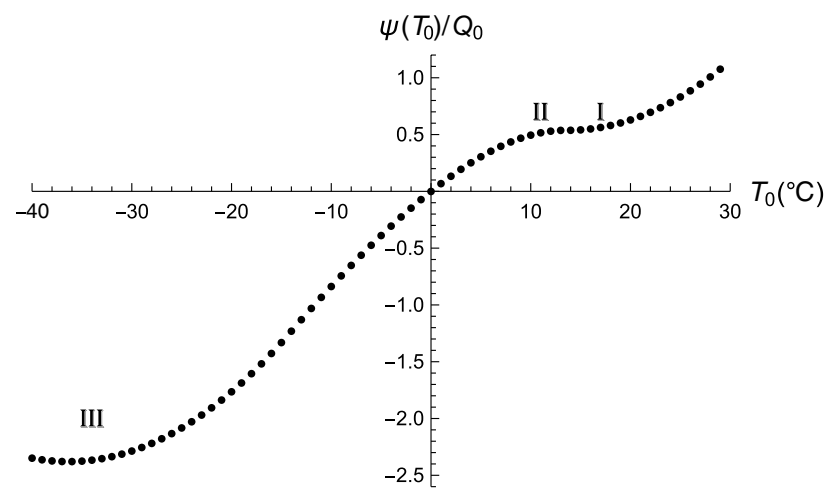

Fig. 10 Trend of the normalized potential function with respect to the global equilibrium temperature $T_{0}$. Labels I, II and III represent the equilibrium states of the climate system, i.e. the current climate condition, an intermediate unstable equilibrium state and the ice-covered state, respectively

Here, $H_{0}\left(T_{0}\right)$ is the planetary co-albedo which is defined by:

$H_{0}\left(x_{s}(T)\right)=a_{i}+\left(a_{w}-a_{i}\right)\left(x_{s}(T)+\frac{1}{2} S_{2}\left(x_{s}(T)-x_{s}(T)^{3}\right)\right)$

where the expression $x_{s}(T)$ represents the ice line given as a function of the temperature for which Eq. (23) is employed with $\delta T$ used as the independent variable.

Solving Eq. (25) for $\lambda$ with $\frac{d Q}{d x_{s}}<0$, the lowest root is found to be $\lambda=-0.3086$. As will be shown shortly, the value found for $\lambda$ determines the reduction of insolation radiation required to drive the climate system from state I to II. Since state II is unstable (as can be seen in Fig. 10) any larger reduction of insolation would make the system fall into state III; therefore, the estimated insolation reduction represents the reduction of insolation required before an ice-covered state is achieved.

In Fig. 11 the lowest root of Eq. (25) is reported for several climate sensitivities and it can be seen that the greater the sensitivity of the model, the closer to zero the value of $\lambda$. The PDE model has a sensitivity of $2.74^{\circ} \mathrm{C}$ for the northern hemisphere, again with $\lambda=-0.3086$. In other cases, $\lambda$ can be less than -1 if the climate model has a sensitivity of $2{ }^{\circ} \mathrm{C}$. Thus, as expected, in a more sensitive model a smaller change in insolation is necessary to reach instability.

Thus, a value of $\lambda$ equal to -0.3086 is now employed to find the control function which would trigger the climate instability and so the solution $T(x, t)$ approaches the icecovered stable solution. Therefore, the result of this investigation provides the limit of SRM in terms of the maximum reduction of insolation before the Earth's climate approaches a new ice-covered state. Again, this provides an extreme operational boundary for SRM, which can be obtained from the analytic PDE model developed in Sect. 2.1. Again, it

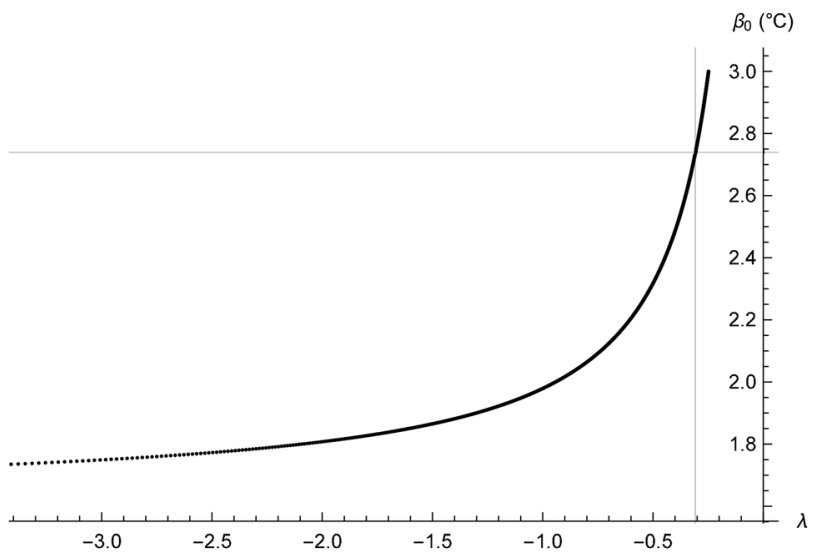

Fig. 11 Values of the lowest root of Eq. (25), i.e. the stability eigenvalue $\lambda$, for several values of the climate sensitivity. The intersection of the grey lines represents the value of $\lambda$ associated to the climate sensitivity of the PDE model

is clearly unlikely that such a control boundary would be reached.

The denominator of Eq. (23) depends on the stability eigenvalue $\lambda$ and can be computed as follow:

$\left(\frac{\partial T(x, \infty)}{\partial x}\right)_{x_{s 0}}=\int_{x_{0}}^{x_{1}} G_{\lambda}\left(x_{s 0}, \xi\right) Q_{0} S(\xi) \frac{\partial \alpha}{\partial x_{s}}\left(\xi, x_{s 0}\right) d \xi$

where $G_{\lambda}$ is a generalization of $G_{x}$ (see Sect. 2.1) and is given as a continuous function of $\lambda$ except at the eigenvalues of $L$ where it is not defined North et al. (1981):

$G_{\lambda}(x, \xi)=\sum_{n=1}^{\infty}(2 n+1) \frac{P(n, x) P(n, \xi)}{L_{n}-\lambda}$

Thus, substituting Eqs. (24) and (29) in Eq. (23), an expression for $\delta x_{s}$ as a function of the control variable $U$ is obtained.

In order to estimate the reduction of solar radiation required to destabilize the climate system and then achieve the condition of an ice-covered state $\left(x_{s} \rightarrow 0\right)$, the quadratic expression in Eq. (31) is considered as a candidate Lyapunov function:

$v\left(x_{s}(x, t, U)\right)=\frac{1}{2} x_{s}(x, t, U)^{2}=\frac{1}{2}\left(x_{s 0}+\delta x_{s}(x, t, U)\right)^{2}$

The Lyapunov stability criterion LaSalle (1976) states that the dynamical system $\dot{x}_{s}=f\left(x_{s}(x, t, U)\right)$ is unstable at $x_{s 0}$ in the sense of Lyapunov if:

(a) $v\left(x_{s 0}\right)=0$

(b) $v\left(x_{s}(x, t, U)\right)>0$ for $x_{s} \neq x_{s 0}$

(c) $\frac{\partial}{\partial t} v\left(x_{s}(x, t, U)\right)>0$ 
In order to satisfy condition (a), function $v\left(x_{s}(x, t, U)\right)$ is computed at $x_{s 0}=0.95$ and the Lyapunov function becomes:

$V\left(x_{s}(x, t, U)\right)=v\left(x_{s}(x, t, U)\right)-v\left(x_{s 0}\right)$

Since the quadratic expression in Eq. (31) is considered, the Lyapunov function is locally positive and therefore condition (b) is satisfied. For condition (c) the first time-derivative of $V$ is developed. Substituting Eq. (23) in Eq. (31), the analytical expression for $V\left(x_{s}(x, t, U)\right)$ can be developed as follow:

$$
\begin{aligned}
V\left(x_{s}(x, t, U)\right)= & U e^{-\frac{\lambda t}{c}} f_{6} \frac{\left(F_{1}(x)\left(f_{5} x_{s 0}-f_{6} T_{0}+F\left(x_{s 0}\right)\left(f_{6} T_{0}-f_{5} x_{s 0}\right)\right)\right)}{f_{5}^{2}} \\
& +\frac{1}{2} U^{2} e^{-\frac{2 \lambda t}{c}} f_{6}^{2} \frac{F_{1}(x)^{2}-F_{1}\left(x_{s 0}\right)^{2}}{f_{5}^{2}}
\end{aligned}
$$

As can be seen in the expression above, $V\left(x_{s}(x, t, U)\right)$ is given by the multiplication of a series of analytical functions which can be found in Appendix A. These expressions are obtained using symbolic computing. In particular, $F_{1}(x)$ depends on the system parameters, such as the planetary albedo, the insolation, the outgoing infra-red radiation, the transport coefficient and the slope of the ice line, whereas, $f_{5}(\lambda)$ and $f_{6}(\lambda)$ depend on the stability eigenvalue $\lambda$. Computing the first time derivative of Eq. (33), which also includes the time-dependence of $U$, and solving the inequality $\frac{\partial}{\partial t} V\left(x_{s}(x, t, U)\right)>0$ for the variable $U$ the following condition is obtained:

$U_{i c e}(x, t) \geq-\frac{f_{U 1}(x)}{f_{U 2}(x)}+\frac{\sqrt{f_{U 2}(x) C_{1}+\frac{f_{U 1}^{2}(x) e^{-\frac{2 \lambda t}{C}}}{f_{U 2}(x)}}}{f_{U 2}(x) \sqrt{\frac{e^{-\frac{2 \lambda t}{c}}}{f_{U 2}(x)}}}$

where the analytical expressions for functions $f_{U 1}(x)$ and $f_{U 2}(x)$ can be found in Appendix B.

As can be seen from Eq. (34), condition (c) of the Lyapunov stability criterion is satisfied by an infinite number of control functions. Each of these functions is identified by a specific value of the parameter $C_{1}$, which enables the selection of the initial condition for the control function $U$. In Fig. 12, control functions with values of $C_{1}$ between 0 and 3 can be found.

If $C_{1}$ is set to zero in Eq. (34), the boundary control function, labelled $U_{\text {bound }}$, is obtained. Combining $U_{\text {bound }}(x, t)$ with the latitudinal distribution of the albedo and the insolation, it is possible to estimate the minimum insolation reduction required to achieve an ice-covered state. Therefore, $U_{\text {bound }}(x, t) S(x) \alpha\left(x, x_{s}\right)$ (see Fig. 13) is the minimum reduction of insolation which would force a transition to an ice-covered equilibrium state. Thus, if the cooling applied is below one of the curves reported in Fig. 12, the current

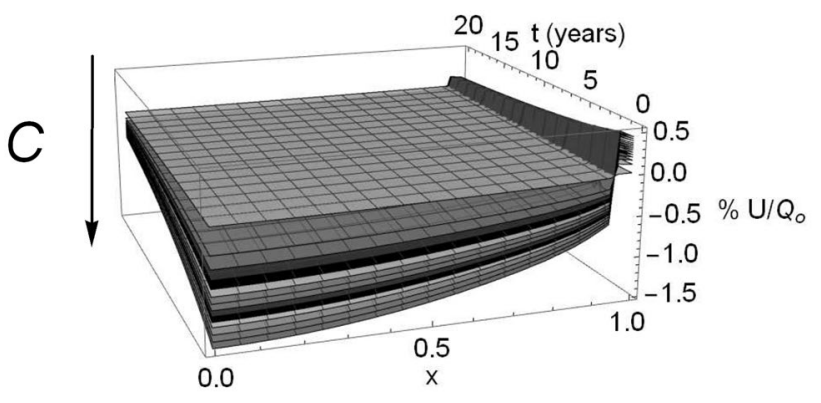

Fig. 12 Family of control $\left(0<C_{1}<3\right)$ functions satisfying condition in Eq. (34)

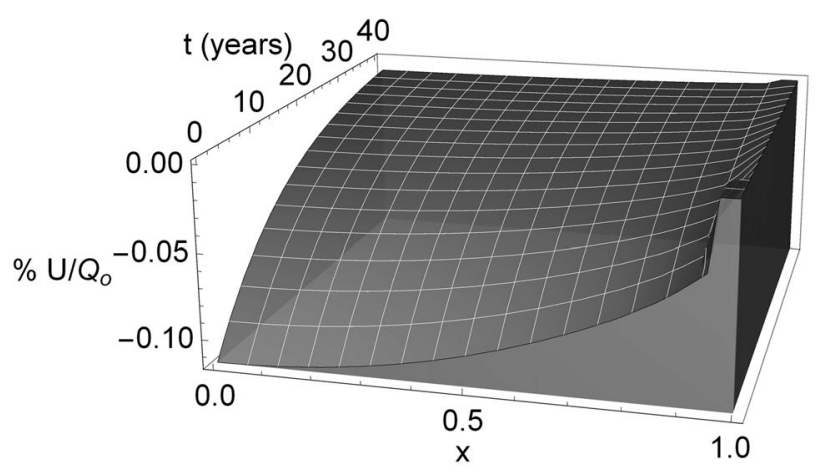

Fig. 13 Boundary control function obtained setting $C_{1}=0$ in Eq. (34), where any SRM profile within the surface which satisfies Eq. (34) will lead to an ice-covered state

climate will converge to the new ice-covered equilibrium state. Considering the boundary function $U_{\text {bound }}(x, t)$, as expected, the required insolation reduction is larger at the equator $\left(U / Q_{0} \simeq-11.5 \%\right.$ for $\left.x=0\right)$ than at high latitudes $\left(U / Q_{0} \simeq-4.16 \%\right.$ for $\left.x=0.90\right)$ and in particular $U / Q_{0}=0$ for $x \geq 0.95$ since that region is already covered by ice. Considering $U_{\text {bound }}(x, t)$ at $t=0$, it is estimated that a minimum overall reduction of insolation of approximately $8.8 \%$ is required to achieve an unstable state (equilibrium condition II in Fig. 10) with $x_{s 0}=0.55$. This result is consistent with the literature Coakley (1979), Caldeira and Kasting (1992), Griffel and Drazin (1981), for example Fig. 3 in Coakley (1979), where it can be seen that a decrease of $8-9 \%$ of the solar constant is required to achieve $x_{s} \simeq 0.5$.

As seen in Fig. 8, in order to counteract a doubling of $\mathrm{CO}_{2}$, a reduction of insolation of $4-4.5 \%$ is required at the poles and only $1-1.5 \%$ at the equator. Comparing this result with Fig. 13, although the cooling required at the equator to achieve the ice-covered state is considerably higher than $1.5 \%$, the required deployment of SRM at the poles to counteract $F_{2 x \mathrm{CO}_{2}}$ is in principle sufficient to move the ice line to lower latitudes. However, if the energy input to the tropics is left nearly constant, as in this case, changes in the albedo of 
the middle and upper latitudes can eventually be mitigated by the exporting of energy from the tropics Schneider and GalChen (1973). Therefore, the instability of the system would not be triggered. This analysis is of importance to climate engineering involving SRM because it demonstrates that the extent of the insolation reduction commonly considered for SRM is rather far from such a catastrophic boundary.

In order to compute the new temperature profile, representing the third equilibrium state (see Fig. 10), a small perturbation $\delta U$ with $\lambda<-1$ is considered. The perturbed temperature obtained by the reduction of insolation given by $U_{\text {bound }}(x, t)$ to drive the system from the current climate state (I) to the unstable state (II) can therefore be computed as follow:

$\delta T_{12_{\text {ice }}}(x, t)=\int_{0}^{t} \delta T_{12}(x, \tau) G_{t}(t, \tau) d \tau$

with $\delta T_{12}$ given by Eq. (24), where the expression for $U$ is substituted from $U_{\text {bound }}$ and $G_{t}$ is reported in Eq. (6) in Sect. 2.1.

To describe the transition from state II to an ice-covered state (III), Eq. (35) becomes:

$\delta T_{23_{\text {ice }}}(x, t)=\int_{0}^{t} \delta T_{23}(x, \tau) G_{t}(t, \tau) d \tau$

with $\delta T_{23}$ given by Eq. (24), where the expression for $U$ is substituted from $U_{\text {bound }}(x, t)+\delta U(x, t)$.

Figure 14 shows the latitudinal distribution of $T_{12_{i c e}}(x, t)=$ $T(x, t)+\delta T_{12_{\text {ice }}}(x, t), \quad T_{23_{\text {ice }}}(x, t)=T(x, t)+\delta T_{23_{\text {ice }}}(x, t)$ and $T(x, t)$, which is given by the equilibrium solution describing the current climate (Eq. (5)). $T_{12_{i c e}}$ and $T_{23_{i c e}}$ represent the equilibrium temperatures reached after the perturbations $\delta T_{12_{\text {ice }}}$ and $\delta T_{23_{\text {ice }}}$ are applied to the system. This latter achieves the climate state III, i.e. the condition of an icecovered Earth with $x_{s}=0$. However, for any $U(x, t)$ smaller than $U_{\text {bound }}(x, t)$ the climate system would remain in state I (current climate with $x_{s 0}=0.95$ ).

In accordance with the literature Gates (1976), in this new climate state the maximum temperature obtained is $-20.44{ }^{\circ} \mathrm{C}$, whereas the global average temperature given by the integration over latitude of $T(x, \infty)$, i.e. the equilibrium temperature, is $-31.77^{\circ} \mathrm{C}$, as expected from the investigation of the potential function for state III (see Fig. 10). This result can be also seen in Fig. 15, where the contours show equilibrium temperatures between $-30^{\circ} \mathrm{C}$ and $-35^{\circ} \mathrm{C}$ for $x_{s} \leq 0.4$ and specific values of $\left(Q_{0}+U\right) / Q_{0}$. As seen in Fig. 15, another important feature for the investigation of the dynamics of the ice line is the trend of $x_{s}$ for a given change of insolation $\left(\left(Q_{0}+U\right) / Q_{0}\right)$. This can be obtained through Eq. (50) in North et al. (1981) where the parameters of the PDE model, averaged between the southern and northern hemisphere in order to better imitate the diffusive model in North et al. (1981), are considered.

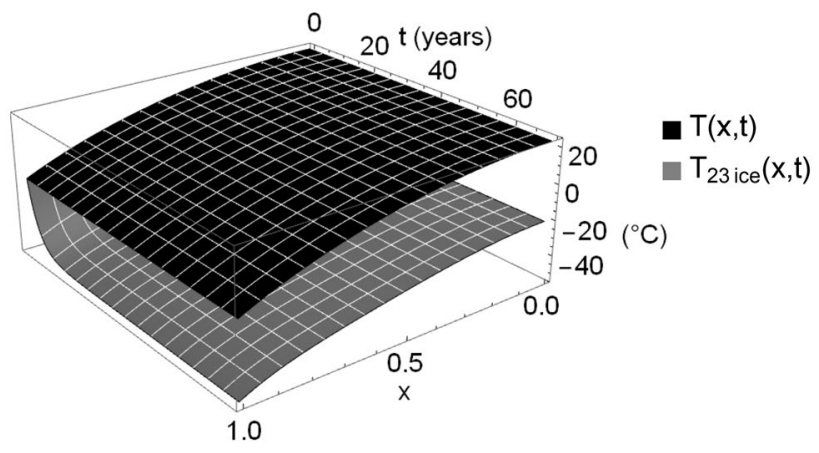

Fig. 14 Trend of the equilibrium temperature of the current climate $(T(x, t))$ and the equilibrium temperatures obtained after the perturbation $U_{\text {bound }}(x, t)+\delta U(t)$ is applied to the system, respectively

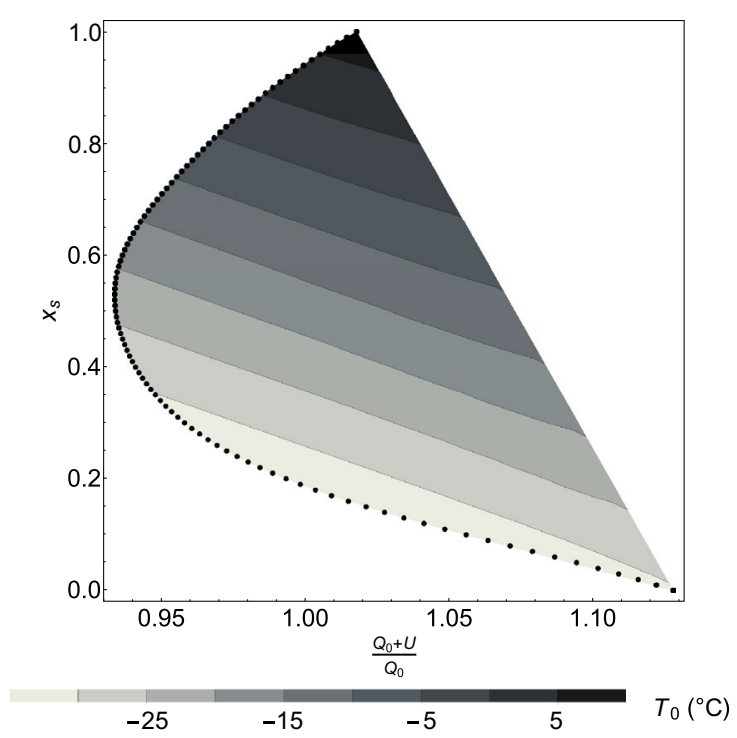

Fig. 15 Dotted curve represents the trend of the position of the ice line $x_{s}$ with the variation of the insolation (normalized over the current value of the solar constant $Q_{0}$ ). The values of $x_{s}$ are obtained computing Eq. (50) in North et al. (1981) with the parameters of the PDE model (see Sect. 2.1). The contours give information on the equilibrium temperature for a given insolation $\left(\left(Q_{0}+U\right) / Q_{0}\right)$ and ice line position $\left(x_{s}\right)$

Despite the differences between the PDE model and the model described in North et al. (1981), such as the overall climate sensitivity and the parametrization of the albedo [in North et al. (1981) a step function is employed for $\alpha\left(x, x_{s}\right)$ ], the results, reported in Fig. 15 of this paper and in Fig. 8 in North et al. (1981), are broadly comparable. In both cases the climate system shows two equilibrium states for current insolation conditions. As can be seen from Fig. 15, if the solar constant is decreased to 0.94 , the unstable equilibrium state is reached and although the solar constant is increased again the ice line decreases further and the ice-covered state is reached. As it will be seen later, a much larger warming 
perturbation is required to lead the system back to the current climate state if an ice-covered state is reached. This outcome is in agreement with the literature where steadystate climate models are considered and suggests that only a $6 \%$ reduction of insolation is required to trigger the instability. Otherwise following the approach developed in this paper (Sect. 5), with the PDE model in Sect. 2.1, it is found that the overall reduction needs to be $8.8 \%$ to make the system unstable (Sect. 4). This discrepancy is due to the timedependency of the PDE model employed Schneider and Gal-Chen (1973).

The change of sign of the curve for $x_{s}>0.8$ in North et al. (1981) is an artefact of the mathematical form of the albedo step function, as explained by North et al. in North et al. (1981), and it is therefore not visible in Fig. 15 because a smooth function is considered for the albedo. Another similar model, where the same function in Eq. (4) is employed for the albedo, can be found in Widiasih (2013). In particular, in Widiasih (2013), the graph of Fig. 15 is obtained through numerical simulations [and can be found in Fig. (4.2) of Widiasih (2013)]. Although the models employ different system parameters, the values of $Q$ are broadly comparable with the normalized values shown in Fig. 15.

Finally, the recovery from an ice-covered state is investigated for completeness. The procedure described in Sect. 5 is applied again in order to estimate the variation of insolation necessary to drive the Earth's climate from an icecovered state (III) to the previous state (the current climate state I). Therefore, in this case, $x_{s 0}=0, T_{s}=-20.44^{\circ} \mathrm{C}$ and $\lambda=-0.28$ [obtained through Eq. (25)] are employed. Again, a family of control functions which would trigger a recovery is found. In Fig. 16, several control functions are reported for values of $C_{1}$ between 0 and 3 . As before, the minimum control function can be found setting $C_{1}=0$ and Fig. 17 is obtained.

Thus, considering the minimum control function reported in Fig. 17, it is estimated that an overall increase of $U / Q_{0}=30 \%$ is required to move the ice line from $x_{s}=0$ back to $x_{s}=0.95$. In particular, a maximum increase of $30.1 \%$ is required at the equator and a minimum of $10.6 \%$ at the pole. This result can be also found in Caldeira and Kasting (1992).

The family of functions $U_{r}(x, t)$ ( $r$ stands for recovery) provides the distribution of insolation increase required to move the climate system back to current conditions. In particular, $U_{r}(x, t)$ is obtained considering Eq. (23) and applying the Lyapunov stability criterion as for the previous case. As before, the new equilibrium temperature can be computed through Eq. (35) and Fig. 18 is then obtained. In particular, the trend of the temperature of the ice-covered climate state is given by $T_{23 i c e}$ whereas the new climate state reached is represented by $T_{\text {rec }}$. It can be noted that the value of the equilibrium temperature at $x=0.95$ is $-10^{\circ} \mathrm{C}$ and the overall equilibrium temperature of the new climate state is $24.1^{\circ} \mathrm{C}$.

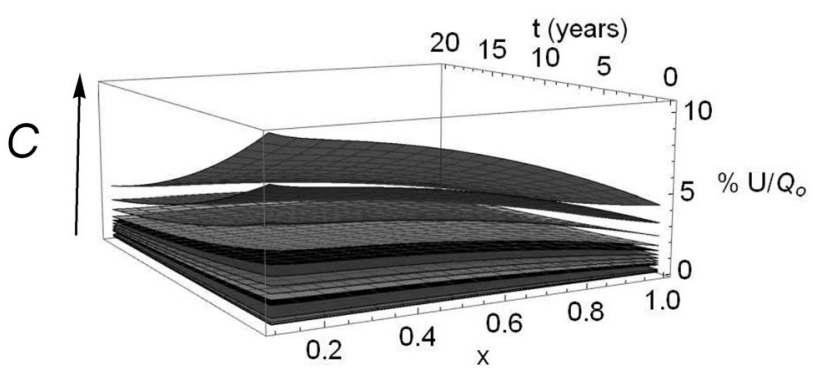

Fig. 16 Family of control functions $\left(0<C_{1}<3\right)$ for the recovery from an ice-covered state

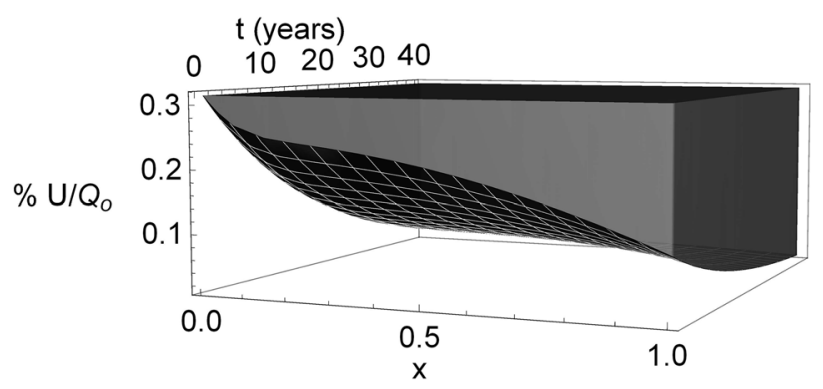

Fig. 17 Minimum control function required to recover from an icecovered state obtained setting $C_{1}=0$, where any SRM profile within the surface, which is also part of the family of functions in Fig. 16, will lead to the current climate state

In accordance with other results from the literature North et al. (1981), the new equilibrium state is found to be much warmer than the previous state with $T \simeq 13^{\circ} \mathrm{C}$ despite that the ice line is at $x_{s 0}=0.95$ in both cases. This is due to latitudinal diffusion of heat towards the poles and the strong ice-albedo feedback. Because of these phenomena, a considerable increase of solar radiation is required near the equator to move the ice line back to the pole and this causes a resulting warmer equilibrium climate state.

\section{Conclusions}

A time-dependent analytical model for the climate system with latitudinal resolution has been developed to assess closed-loop climate engineering strategies. The system investigated is a PDE model which can be analytically solved for any external forcing providing the latitudinal distribution of the temperature perturbation with time.

The model can be employed to investigate climate engineering strategies taking into account latitudinal disparities. High-fidelity numerical models for the climate can also be used to evaluate climate engineering strategies, but these models are computationally expensive. In contrast, the use of the PDE model provides a useful tool to rapidly asses SRM 


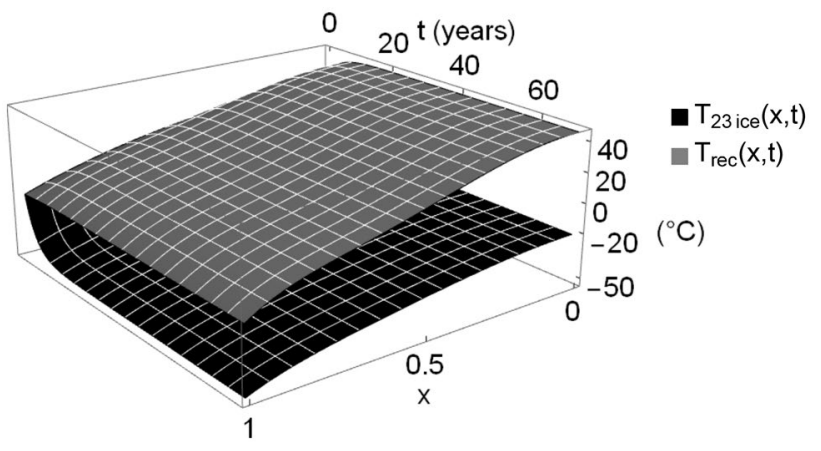

Fig. 18 Trend of the temperature of the ice-covered climate state $\left(T_{23 i c e}(x, t)\right)$ and the temperature obtained after the perturbation $U_{r}(x, t) S(x) \alpha\left(x, x_{s 0}\right)$ is applied to the system, which is given by $T_{\text {rec }}(x, t)$

strategies, providing a clear understanding of the climate dynamics involved.

The PDE model developed in this paper is employed in three simulations [cases (1), (2), (3)] to explore multi-objective strategies with a PI feedback control. Several objectives were simultaneously minimized and the latitudinal response investigated considering a steady increase of $\mathrm{CO}_{2}$ concentration in the atmosphere ( $1 \mathrm{pctCO}_{2}$ scenario). The model provided analytical expressions for suitable control functions for three strategies, and proved to be effective when multiobjective analyses are considered. In fact, despite the simplicity of the model, results, which are broadly comparable with the literature, are found. The distributions of the control functions with latitude are obtained using the analytical solution of the PDE model and are again consistently comparable with the literature. In particular, the control responses and the temperature trends obtained with the analytical solution of the PDE model are comparable with those achieved through the CESM 1.0.2 (Community Earth System Model). This approach is considered as the verification of the general correctness and usefulness of the model developed.

Moreover, the model is used for the analysis of the zonal mean temperature. In agreement with the literature, it is found that the rms zonal mean temperature anomaly caused by the $1 \mathrm{pctCO}_{2}$ scenario decreases when more degrees of freedom are managed. Also, the PDE model can be employed to find the exact control law required and reduce the zonal mean temperature perturbation to zero. It is found that a nonuniform SRM distribution with increased control closer to the poles provides a more uniform offsetting of $\mathrm{CO}_{2}$-induced warming and restores the mean temperature without overcooling equatorial regions and under-cooling polar regions.

As a further example of the application of the PDE model, the upper limit on SRM is investigated through the analysis of the dynamics of the ice line. This analysis is of importance for climate engineering involving SRM because it shows that the extent of the insolation reduction commonly considered for SRM is far from the insolation reduction required to trigger instability of the climate. With respect to other similar calculations in the literature, where the insolation reduction required to achieve an ice-covered state is estimated, in this case, the whole family of control functions which would destabilise the system is found and analytical expressions are provided as a function of both latitude and time.

In this context, it is found that the minimum overall control effort required for an ice-covered state is approximately $8.8 \%$, which decreases towards zero as the climate cools. In particular, the maximum insolation reduction is needed at the equator $(11.5 \%)$ and the minimum at high latitudes (4.2\%). The system then falls into a stable ice-covered state, where the global equilibrium temperature is estimated to be $-32^{\circ} \mathrm{C}$.

The use of the PDE model allows a clear and quick assessment of the boundaries of SRM, proving that the insolation reduction required to move the ice line to the equator is much higher than that considered for SRM deployment. It is therefore highly unlikely to accidentally force an ice-covered state.

Acknowledgements Federica Bonetti acknowledges a University of Glasgow PhD scholarship and Colin McInnes acknowledges support from a Royal Society Wolfson Research Merit Award.

Open Access This article is distributed under the terms of the Creative Commons Attribution 4.0 International License (http://creativeco mmons.org/licenses/by/4.0/), which permits unrestricted use, distribution, and reproduction in any medium, provided you give appropriate credit to the original author(s) and the source, provide a link to the Creative Commons license, and indicate if changes were made.

\section{Appendices}

\section{Appendix A: Analytical functions of $V\left(x_{s}(U, t)\right)$}

As seen in Sect. 5, the Lyapunov function depends on a number of analytical functions (Eq. 33) depending on the model parameters, the stability eigenvalue and the slope of the ice line. Their complete description is given below. In particular, function $F_{1}(x)$ is given by the combination of several functions as reported in Eq. (37):

$F_{1}(x)=Q_{0} \frac{\sqrt{f_{d 1}} a_{w} F_{w}(x)+\frac{a_{w}^{2} \Theta_{\alpha} F_{w_{2}}(x)+a_{i}^{2} \Theta_{\alpha} F_{i}(x)+a_{i}\left(F_{i_{2}}(x)+a_{w} F_{w_{3}}(x) \Theta_{\alpha}\right)}{\left(B^{2}+62 B D+840 D^{2}\right)}}{f_{d_{1}}}$

where the functions $\Theta_{\alpha}, f_{d_{1}}, F_{w}(x), F w_{2}(x), F w_{3}(x), F_{i}(x)$ and $F_{i_{2}}(x)$ are given by the following expressions:

$\Theta_{\alpha}=\frac{d x_{s}}{d Q}\left(x_{s 0}\right) \frac{Q_{0}}{(S \alpha)_{x_{s 0}}}$

$f_{d_{1}}=B(B+6 D)(B+20 D)(B+42 D)$ 


$$
\begin{aligned}
F_{w}(x)= & B^{3}\left(0.027 x^{6}-1.0 x^{4}+0.25 x^{2}+1.0\right)+B^{2} D\left(0.71 x^{6}-49 x^{4}+2.8 x^{2}+68\right) \\
& +S_{2}\left(B^{3}\left(-1.3 x^{6}+0.57 x^{4}+1.5 x^{2}-0.50\right)+B^{2} D\left(-34 x^{6}-12 x^{4}+98 x^{2}-31\right)\right. \\
& \left.+B D^{2}\left(-160 x^{6}-90 x^{4}+1000 x^{2}-410\right)-420 D^{3}\right)+B D^{2}\left(3.3 x^{6}-260 x^{4}-310 x^{2}\right. \\
& +1200)+4400 D^{3}
\end{aligned}
$$

$$
\begin{aligned}
F_{w_{2}}(x)= & B^{8}\left(-6.3 x^{6}+9.1 x^{4}-1.8 x^{2}+0.057\right)+B^{7} D\left(-1000 x^{6}+1500 x^{4}\right. \\
& \left.-250 x^{2}+7.9\right)+B^{6} D^{2}\left(-66000 x^{6}+98000 x^{4}-12000 x^{2}+490\right)+B^{5} D^{3} \\
& \left(-2.3 \times 10^{6} x^{6}+3.4 \times 10^{6} x^{4}-210000 x^{2}+21000\right)+B^{4} D^{4}\left(-4.3 \times 10^{7} x^{6}\right. \\
& \left.+6.6 \times 10^{7} x^{4}+1.8 \times 10^{6} x^{2}+830000\right)+B^{3} D^{5}\left(-4.6 \times 10^{8} x^{6}+7.1 \times 10^{8} x^{4}\right. \\
& \left.+1.2 \times 10^{8} x^{2}+2.4 \times 10^{7}\right)+B^{2} D^{6}\left(-2.5 \times 10^{9} x^{6}+3.9 \times 10^{9} x^{4}+1.4 \times 10^{9} x^{2}\right. \\
& \left.+4.3 \times 10^{8}\right)+S_{2}\left(B^{8}\left(-6.1 x^{6}+11 x^{4}-2.5 x^{2}+0.083\right)+B^{7} D\left(-920 x^{6}+1700 x^{4}\right.\right. \\
& \left.-360 x^{2}+11\right)+B^{6} D^{2}\left(-56000 x^{6}+110000 x^{4}-19000 x^{2}+620\right)+B^{5} D^{3} \\
& \left(-1.7 \times 10^{6} x^{6}+3.5 \times 10^{6} x^{4}-420000 x^{2}+21000\right)+B^{4} D^{4}\left(-2.8 \times 10^{7} x^{6}\right. \\
& \left.+6.2 \times 10^{7} x^{4}-2.1 \times 10^{6} x^{2}+670000\right)+B^{3} D^{5}\left(-2.4 \times 10^{8} x^{6}+6.0 \times 10^{8} x^{4}\right. \\
& \left.+6.3 \times 10^{7} x^{2}+1.9 \times 10^{7}\right)+B^{2} D^{6}\left(-8.6 \times 10^{8} x^{6}+2.7 \times 10^{9} x^{4}+8.8 \times 10^{8} x^{2}\right. \\
& \left.+3.2 \times 10^{8}\right)+S_{2}\left(B^{8}\left(-1.2 x^{6}+2.9 x^{4}-0.89 x^{2}+0.030\right)+B^{7} D\left(-170 x^{6}+460 x^{4}\right.\right. \\
& \left.-130 x^{2}+3.9\right)+B^{6} D^{2}\left(-10000 x^{6}+29000 x^{4}-7100 x^{2}+190\right)+B^{5} D^{3}\left(-290000 x^{6}\right. \\
& \left.+910000 x^{4}-170000 . x^{2}+4500\right)+B^{4} D^{4}\left(-4.4 \times 10^{6} x^{6}+1.5 \times 10^{7} x^{4}-1.0 \times 10^{6} x^{2}\right. \\
& +110000)+B^{3} D^{5}\left(-3.1 \times 10^{7} x^{6}+1.1 \times 10^{8} x^{4}+1.7 \times 10^{7} x^{2}+3.6 \times 10^{6}\right)+B^{2} D^{6} \\
& \left(-5.5 \times 10^{7} x^{6}+2.1 \times 10^{8} x^{4}+1.9 \times 10^{8} x^{2}+6.9 \times 10^{7}\right)+B D^{7}\left(1.3 \times 10^{8} x^{6}\right. \\
& \left.\left.-5.0 \times 10^{8} x^{4}-2.8 \times 10^{8} x^{2}+4.5 \times 10^{8}\right)-7.0 \times 10^{8} D^{8}\right)+B D^{7}\left(-8.7 \times 10^{8} x^{6}+4.4 \times 10^{9} x^{4}\right. \\
& \left.\left.+2.3 \times 10^{9} x^{2}+2.6 \times 10^{9}\right)+5.9 \times 10^{9} D^{8}\right)+B D^{7}\left(-5.3 \times 10^{9} x^{6}+8.3 \times 10^{9} x^{4}+5.8 \times 10^{9} x^{2}\right. \\
& \left.+3.9 \times 10^{9}\right)+1.4 \times 10^{10} D^{8}
\end{aligned}
$$

$$
\begin{aligned}
F_{w_{3}}(x)= & B^{8}\left(S _ { 2 } \left(S_{2}\left(0.076 x^{6}-0.19 x^{4}+0.059 x^{2}-0.0020\right)+0.43 x^{6}-0.76 x^{4}+0.19 x^{2}\right.\right. \\
& \left.-0.0061)+0.51 x^{6}-0.75 x^{4}+0.15 x^{2}-0.0047\right)+B^{7} D\left(S _ { 2 } \left(S_{2}\left(8.4 x^{6}-23 x^{4}+6.3 x^{2}-0.18\right)\right.\right. \\
& \left.\left.+70 x^{6}-150 x^{4}+37 x^{2}-1.2\right)+150 x^{6}-220 x^{4}+39 x^{2}-1.2\right)+B^{6} D^{2}\left(S _ { 2 } \left(S _ { 2 } \left(330 x^{6}-980 x^{4}\right.\right.\right. \\
& \left.\left.\left.+210 x^{2}-5.0\right)+5100 x^{6}-12000 x^{4}+3000 x^{2}-92\right)+15000 x^{6}-22000 x^{4}+3200 x^{2}-110\right)+B^{5} D^{3} \\
& \left(S_{2}\left(S_{2}\left(2300 x^{6}-10000 x^{4}-730 x^{2}+7.6\right)+190000 x^{6}-550000 x^{4}+120000 x^{2}-3600\right)\right. \\
& \left.+710000 x^{6}-1.1 \times 10^{6} x^{4}+110000 x^{2}-5600\right)+B^{4} D^{4}\left(S _ { 2 } \left(S _ { 2 } \left(-220000 x^{6}+590000 x^{4}\right.\right.\right. \\
& \left.\left.-220000 x^{2}+1800\right)+3.9 \times 10^{6} x^{6}-1.4 \times 10^{7} x^{4}+2.2 \times 10^{6} x^{2}-83000\right)+1.8 \times 10^{7} x^{6}-2.8 \times 10^{7} x^{4} \\
& \left.+640000 x^{2}-230000\right)+B^{3} D^{5}\left(S _ { 2 } \left(S_{2}\left(-7.1 \times 10^{6} x^{6}+2.3 \times 10^{7} x^{4}-4.3 \times 10^{6} x^{2}-7600\right)\right.\right. \\
& \left.+3.9 \times 10^{7} x^{6}-1.8 \times 10^{8} x^{4}+9.8 \times 10^{6} x^{2}-2.0 \times 10^{6}\right)+2.5 \times 10^{8} x^{6}-3.9 \times 10^{8} x^{4}-3.8 \times 10^{7} x^{2} \\
& \left.-8.0 \times 10^{6}\right)+B^{2} D^{6}\left(S _ { 2 } \left(S_{2}\left(-7.9 \times 10^{7} x^{6}+2.8 \times 10^{8} x^{4}+5.0 \times 10^{6} x^{2}+1.2 \times 10^{6}\right)\right.\right. \\
& \left.+1.5 \times 10^{8} x^{6}-1.2 \times 10^{9} x^{4}-1.6 \times 10^{8} x^{2}-5.1 \times 10^{7}\right)+1.8 \times 10^{9} x^{6}-2.7 \times 10^{9} x^{4}-8.2 \times 10^{8} x^{2} \\
& \left.-1.9 \times 10^{8}\right)+B D^{7}\left(S _ { 2 } \left(S_{2}\left(-2.7 \times 10^{8} x^{6}+9.9 \times 10^{8} x^{4}+5.5 \times 10^{8} x^{2}+1 \times 10^{8}\right)\right.\right. \\
& \left.+1.5 \times 10^{8} x^{6}-2.8 \times 10^{9} x^{4}-1.3 \times 10^{9} x^{2}-7.2 \times 10^{8}\right)+4.5 \times 10^{9} x^{6}-7.0 \times 10^{9} x^{4}-4.9 \times 10^{9} x^{2} \\
& \left.-2.4 \times 10^{9}\right)+D^{8}\left(S_{2}\left(1.4 \times 10^{9} S_{2}-3.5 \times 10^{9}\right)-1.2 \times 10^{10}\right)
\end{aligned}
$$




$$
\begin{aligned}
& F_{i}(x)=B^{8}\left(5.8 x^{6}-8.4 x^{4}+1.7 x^{2}-0.052\right)+B^{7} D\left(860 x^{6}-1300 x^{4}+210 x^{2}-6.7\right) \\
& +B^{6} D^{2}\left(51000 x^{6}-76000 x^{4}+9000 x^{2}-370\right)+B^{5} D^{3}\left(1.5 \times 10^{6} x^{6}-2.3 \times 10^{6} x^{4}\right. \\
& \left.+110000 x^{2}-16000\right)+B^{4} D^{4}\left(2.5 \times 10^{7} x^{6}-3.8 \times 10^{7} x^{4}-2.5 \times 10^{6} x^{2}-600000\right) \\
& +B^{3} D^{5}\left(2.1 \times 10^{8} x^{6}-3.2 \times 10^{8} x^{4}-7.7 \times 10^{7} x^{2}-1.6 \times 10^{7}\right)+B^{2} D^{6} \\
& \left(7.5 \times 10^{8} x^{6}-1.2 \times 10^{9} x^{4}-6.2 \times 10^{8} x^{2}-2.4 \times 10^{8}\right)+S_{2}\left(B ^ { 8 } \left(5.6 x^{6}-9.8 x^{4}\right.\right. \\
& \left.+2.4 x^{2}-0.077\right)+B^{7} D\left(850 x^{6}-1500 x^{4}+320 x^{2}-10\right)+B^{6} D^{2}\left(51000 x^{6}-94000 x^{4}\right. \\
& \left.+16000 x^{2}-530\right)+B^{5} D^{3}\left(1.5 \times 10^{6} x^{6}-2.9 \times 10^{6} x^{4}+300000 x^{2}-18000\right)+B^{4} D^{4} \\
& \left(2.4 \times 10^{7} x^{6}-4.9 \times 10^{7} x^{4}-61000 x^{2}-590000\right)+B^{3} D^{5}\left(2.0 \times 10^{8} x^{6}-4.1 \times 10^{8} x^{4}\right. \\
& \left.-7.3 \times 10^{7} x^{2}-1.7 \times 10^{7}\right)+B^{2} D^{6}\left(7.1 \times 10^{8} x^{6}-1.5 \times 10^{9} x^{4}-7.2 \times 10^{8} x^{2}-2.7 \times 10^{8}\right) \\
& +S_{2}\left(B^{8}\left(1.1 x^{6}-2.7 x^{4}+0.83 x^{2}-0.028\right)+B^{7} D\left(160 x^{6}-430 x^{4}+120 x^{2}-3.8\right)\right. \\
& +B^{6} D^{2}\left(9700 x^{6}-28000 x^{4}+6900 x^{2}-180\right)+B^{5} D^{3}\left(290000 x^{6}-900000 x^{4}\right. \\
& \left.+170000 x^{2}-4500\right)+B^{4} D^{4}\left(4.7 \times 10^{6} x^{6}-1.5 \times 10^{7} x^{4}+1.3 \times 10^{6} x^{2}-110000\right) \\
& +B^{3} D^{5}\left(3.8 \times 10^{7} x^{6}-1.3 \times 10^{8} x^{4}-1.3 \times 10^{7} x^{2}-3.6 \times 10^{6}\right)+B^{2} D^{6}\left(1.3 \times 10^{8} x^{6}\right. \\
& \left.-4.9 \times 10^{8} x^{4}-2.0 \times 10^{8} x^{2}-7.1 \times 10^{7}\right)+B D^{7}\left(1.3 \times 10^{8} x^{6}-5.0 \times 10^{8} x^{4}-2.8 \times 10^{8} x^{2}\right. \\
& \left.\left.-5.5 \times 10^{8}\right)-7.0 \times 10^{8} D^{8}\right)+B D^{7}\left(7.2 \times 10^{8} x^{6}-1.6 \times 10^{9} x^{4}-9.9 \times 10^{8} x^{2}-1.9 \times 10^{9}\right) \\
& \left.-2.4 \times 10^{9} D^{8}\right)+B D^{7}\left(8.0 \times 10^{8} x^{6}-1.3 \times 10^{9} x^{4}-8.7 \times 10^{8} x^{2}-1.5 \times 10^{9}\right)-2.0 \times 10^{9} D^{8} \\
& F_{i_{2}}(x)=B^{9}\left(S_{2}\left(1.3 x^{6}-0.57 x^{4}+0.035 x^{2}+0.00018\right)-0.027 x^{6}+1.0 x^{4}-0.25 x^{2}\right. \\
& +0.0091)+B^{8} D\left(S_{2}\left(200 x^{6}-62 x^{4}-0.071 x^{2}+0.11\right)-4.2 x^{6}+180 x^{4}-35 x^{2}+1.3\right) \\
& +B^{7} D^{2}\left(S_{2}\left(13000 x^{6}-1900 x^{4}-170 x^{2}+2.8\right)-260 x^{6}+13000 x^{4}-1600 x^{2}+78\right) \\
& +B^{6} D^{3}\left(S_{2}\left(410000 x^{6}+7300 x^{4}+3200 x^{2}-210\right)-8600 x^{6}+490000 x^{4}-12000 x^{2}\right. \\
& +3400)+B^{5} D^{4}\left(S_{2}\left(7.3 \times 10^{6} x^{6}+1.4 \times 10^{6} x^{4}+730000 x^{2}+9700\right)-150000 x^{6}\right. \\
& \left.+9.8 \times 10^{6} x^{4}+1.2 \times 10^{6} x^{2}+150000\right)+B^{4} D^{5}\left(S _ { 2 } \left(7.2 \times 10^{7} x^{6}+2.6 \times 10^{7} x^{4}+2.3 \times 10^{7} x^{2}\right.\right. \\
& \left.\left.+1.5 \times 10^{6}\right)-1.5 \times 10^{6} x^{6}+1.1 \times 10^{8} x^{4}+3.7 \times 10^{7} x^{2}+5.1 \times 10^{6}\right)+B^{3} D^{6}\left(S _ { 2 } \left(3.5 \times 10^{8} x^{6}\right.\right. \\
& \left.\left.+1.7 \times 10^{8} x^{4}+2.6 \times 10^{8} x^{2}+4.6 \times 10^{7}\right)-7.3 \times 10^{6} x^{6}+5.5 \times 10^{8} x^{4}+4.0 \times 10^{8} x^{2}+9.8 \times 10^{7}\right) \\
& +B^{2} D^{7}\left(S_{2}\left(6.6 \times 10^{8} x^{6}+3.8 \times 10^{8} x^{4}+8.9 \times 10^{8} x^{2}+5.2 \times 10^{8}\right)-1.4 \times 10^{7} x^{6}\right. \\
& \left.+1.1 \times 10^{9} x^{4}+1.3 \times 10^{9} x^{2}+9.0 \times 10^{8}\right)+B D^{8}\left(1.8 \times 10^{9} S_{2}+2.8 \times 10^{9}\right)
\end{aligned}
$$

Finally, $f_{5}(\lambda)$ and $f_{6}(\lambda)$ depend on the stability eigenvalue and are given by the numerator and denominator of Eq. (29), respectively.

$$
\begin{aligned}
f_{5}(\lambda)= & 1.6 Q_{0}\left(a_{i}-a_{w}\right)\left(\lambda \left(\lambda\left(\lambda\left(S_{2}+1.5\right)-44 S_{2}-66\right)\right.\right. \\
& \left.\left.+490 S_{2}+750\right)-1000 S_{2}-1700\right) \\
f_{6}(\lambda)= & (\lambda-29)(\lambda-15)(\lambda-5.5)(\lambda-1.6)
\end{aligned}
$$$$
\text { Appendix B: Analytical functions of } U_{\text {ice }}(x, t)
$$

The expression found in Eq. (34) for the family of control functions which would trigger the instability of the climate system depend on functions $f_{U 1}$ and $f_{U 2}$. These functions are given below:

$$
f_{U 1}(x)=\frac{f_{5} x_{s 0} F_{1}\left(x_{s 0}\right)-f_{5} x_{s 0} F_{1}(x)-f_{6} F_{1}\left(x_{s 0}\right) T_{0}+f_{6} F_{1}(x) T_{0}}{1.30 \times 10^{6}}
$$

$$
f_{U 2}(x)=\frac{\left(F_{1}^{2}\left(x_{s 0}\right)-F_{1}^{2}(x)\right)^{2}}{76434.12}
$$

\section{References}

Bala Govindasamy, Duffy PB, Taylor KE (2008) Impact of geoengineering schemes on the global hydrological cycle. In: Proceedings 
of the National Academy of Sciences. vol 105, National Acad Sciences, number 22, pp 7664-7669

Ban-Weiss GA, Caldeira K (2010) Geoengineering as an optimization problem. Environ Res Lett 5(3):034,009. https://doi. org/10.1088/1748-9326/5/3/034009

Blackstock JJ, Battisti DS, Caldeira K, Eardley DM, Katz JI, Keith DW, Patrinos AA, Schrag DP, Socolow RH, Koonin SE (2009) Climate engineering responses to climate emergencies, IOP conference series: earth and environmental science. IOP Earth Environ Sci. https://doi.org/10.1088/1755-1307/6/5/452015

Budyko MI (1969) The effect of solar radiation variations on the climate of the Earth. Tellus 21(5):611-619. https://doi. org/10.1111/j.2153-3490.1969.tb00466.x

Bonetti F, McInnes CR (2018) Multiple input control strategies for robust and adaptive climate engineering in a low order 3-box model. In Proceeding A of Royal Society, under review

Caldeira K, Kasting JF (1992) Susceptibility of the early earth to irreversible glaciation caused by carbon dioxide clouds. Nature 359(6392):226. https://doi.org/10.1038/359226a0

Cess RD (1976) Climate change: an appraisal of atmospheric feedback mechanisms employing zonal climatology. J Atmos Sci 33(10):1831-1843

Coakley James A Jr (1979) A study of climate sensitivity using a simple energy balance model. J Atmos Sci 36(2):260-269

Cole KD, Beck JV, Haji-Sheikh A, Litkouhi B (2010) Heat conduction using Greens functions. Taylor and Francis, London

Collins M, Knutti R, Arblaster J, Dufresne J-L, Fichefet T, Friedlingstein P, Gao X, Gutowski WJ, Johns T, Krinner G, Shongwe M, Tebaldi C, Weaver AJ, Wehner M (2013) Long-term climate change: projections, commitments and irreversibility. In: Stocker TF, Qin D, Plattner G-K, Tignor M, Allen SK, Boschung J, Nauels A, Xia Y, Bex V, Midgley PM (eds) Climate change 2013: the physical science basis. Contribution of working group I to the fifth assessment report of the intergovernmental panel on climate change. Cambridge University Press, Cambridge, UK

Dai Z, Weisenstein DK, Keith DW Tailoring meridional and seasonal radiative forcing by sulfate aerosol solar geoengineering. Geophys Res Lett. https://doi.org/10.1002/2017GL076472

Dlugokencky E, Tans P (2016) Trends in atmospheric carbon dioxide: recent global $\mathrm{CO}_{2}$. ESRL Global Monitoring Division Global Greenhouse Gas Reference Network. [online] Esrl.noaa. gov. Available at: https://www.esrl.noaa.gov/gmd/ccgg/trends/. Accessed 5 July 2018

Gasser T, Guivarch C, Tachiiri K, Jones C, Ciais P (2015) Negative emissions physically needed to keep global warming below $2{ }^{\circ} \mathrm{C}$. Nat Commun. https://doi.org/10.1038/ncomms8958

Gates WL (1976) Modeling the ice-age climate. Science 191(4232):1138-1144. https://doi.org/10.1126/scien ce. 191.4232 .1138

Govindasamy B, Caldeira K (2000) Geoengineering Earth's radiation balance to mitigate $\mathrm{CO}_{2}$-induced climate change. Geophys Res Lett 27(14):2141-2144

Govindasamy B, Caldeira K, Duffy P (2003) Geoengineering Earth's radiation balance to mitigate climate change from a quadrupling of $\mathrm{CO}_{2}$. Glob Planet Change 37(1):157-168

Griffel D, Drazin P (1981) On diffusive climatological models. J Atmos Sci 38(11):2327-2332

Hahn David W, Ozisik MN (2012) Heat conduction, 3rd edn. Wiley, Hoboken

Heyen D, Wiertz T, Irvine PJ (2015) Regional disparities in SRM impacts: the challenge of diverging preferences. Clim Change 133:557. https://doi.org/10.1007/s10584-015-1526-8
Kang Sarah M, Seager Richard (2012) Croll revisited: why is the northern hemisphere warmer than the southern hemisphere? Columbia University Academic Commons. https://doi.org/10.7916/D80R9 XNQ

Kravitz B, MacMartin DG, Mills MJ, Richter JH, Tilmes S, Lamarque JF, Tribbia JJ, Vitt F (2017) First simulations of designing stratospheric sulfate aerosol geoengineering to meet multiple simultaneous climate objectives. J Geophys Res Atmos 122(23):1261612634. https://doi.org/10.1002/2017JD026874

Kravitz B, Robock A, Boucher O, Schmidt H, Taylor KE, Stenchikov G, Schulz M (2011) The geoengineering model intercomparison project (GeoMIP). Atmos Sci Lett 12(2):162-167

Kravitz Ben, MacMartin DG, Rasch PJ (2016) Geoengineering as a design problem. Earth Syst Dyn 7(2):469

LaSalle JP (1976) The stability of dynamical systems. In: Regional conference series in appliedmathematics. SIAM, Philadelphia. https://doi.org/10.1137/1021079

MacMartin D, Keith D, Kravitz B, Caldeira K (2012) Managing tradeoffs in geoengineering through optimal choice of non-uniform radiative forcing. Nat Clim Change 3:365-368. https://doi. org/10.1038/nclimate 1722

MacMartin DG, Kravitz B, Keith DW (2014) Geoengineering, The world's largest control problem. In: 2014 American control conference. IEEE, Piscataway, pp 2401-2406. https://doi.org/10.1109/ ACC.2014.6858658

MacMartin DG, Kravitz B, Keith DW, Jarvis A (2014) Dynamics of the coupled human-climate system resulting from closed-loop control of solar geoengineering. Clim Dyn 43(1-2):243-258

MacMartin DG, Kravitz B, Tilmes S, Richter JH, Mills MJ, Lamarque JF, Tribbia JJ, Vitt F (2017) The climate response to stratospheric aerosol geoengineering can be tailored using multiple injection locations. J Geophys Res Atmos 122(23):12574-12590. https:// doi.org/10.1002/2017JD026868

MacMynowski DG, Shin HJ, Caldeira K (2011) The frequency response of temperature and precipitation in a climate model. Geophys Res Lett. https://doi.org/10.1029/2011GL048623

McGuffie K, Henderson-Sellers A (2005) A climate modelling primer, 3rd edn. Wiley, Chichester. https://doi.org/10.1002/0470857617 .ch6

Moreno-Cruz JB, Ricke KL, Keith DW (2012) A simple model to account for regional inequalities in the effectiveness of solar radiation management. Clim Change 110(3):649-668. https:// doi.org/10.1007/s10584-011-0103-z

North GR (1975) Analytical solution to a simple climate model with diffusive heat transport. J Atmos Sci 32(7):1301-1307

North GR, Cahalan RF, Coakley JA (1981) Energy balance climate models. Rev Geophys 19(1):91-121. https://doi.org/10.1029/ RG019i001p00091

Robert F, North GR (1979) A stability theorem for energy-balance climate models. J Atmos Sci 36:1205-1216

Schneider SH, Gal-Chen T (1973) Numerical experiments in climate stability. J Geophys Res 78(27):6182-6194. https://doi. org/10.1029/JC078i027p06182

Sellers WD (1969) A global climatic model based on the energy balance of the earth-atmosphere system. J Appl Meteorol 8(3):392400. https://doi.org/10.1175/1520-0450(1969)008\%3C039 2:AGCMBO\%3E2.0.CO;2

Vaughan NE, Lenton TM (2011) A review of climate geoengineering proposals. Clim Change 109(3-4):745-790. https://doi. org/10.1007/s10584-011-0027-7

Widiasih ER (2013) Dynamics of the budyko energy balance model. SIAM J Appl Dyn Syst 12(4):2068-2092 